\title{
Konzeptualisierung und Diagnostik von Präsentationskompetenz
}

\author{
Evelin Herbein ${ }^{1,2}$ (D) $\cdot$ Fabian Ruth $^{3} \cdot$ Carmen Lipphardt $^{3} \cdot$ Tobias Ringeisen $^{4}$
}

Angenommen: 1. April 2021 / Online publiziert: 4. Mai 2021

(c) Der/die Autor(en) 2021

\section{Zusammenfassung}

Dieser Beitrag in der Zeitschrift „Gruppe. Interaktion. Organisation (GIO)“ stellt einen konzeptionellen Rahmen der Präsentationskompetenz vor, auf dessen Basis ein Überblick zu validierten deutschsprachigen Instrumenten zur Erfassung von Präsentationskompetenz gegeben wird. Dieser umfasst sowohl Fremd- als auch Selbsteinschätzungsverfahren. Trotz der Bedeutsamkeit des Präsentierens sowohl im beruflichen als auch im (hoch)schulischen Kontext liegt bisher keine einheitliche Konzeptualisierung von Präsentationskompetenz vor. Zudem mangelt es an Ansätzen, die die Perspektiven unterschiedlicher Disziplinen, wie der Psychologie oder der Rhetorik, berücksichtigen. Basierend auf existierender Forschung wird mit Hilfe der beiden Disziplinen ein übergeordneter konzeptioneller Rahmen abgeleitet, der einen disziplinübergreifenden und differenzierten Zugriff auf die Präsentationskompetenz ermöglicht und sich auf den gesamten Arbeits- und Organisationskontext übertragen lässt. Den Abschluss des Beitrags bilden Implikationen zur spezifischen Erfassung und bedarfsorientierten Förderungen von Präsentationskompetenz.

Schlüsselwörter Präsentationskompetenz $\cdot$ Kompetenzmodell $\cdot$ Diagnostik $\cdot$ Assessment $\cdot$ Selbsteinschätzung

\section{Conceptualization and assessment of presentation competence}

\begin{abstract}
This article-published in the journal "Gruppe. Interaktion. Organisation (GIO)"-introduces a conceptual framework of presentation competence, by means of which an overview of validated German-language instruments for the assessment of presentation competence is provided. Thereby, external and self-assessment tools are considered. Despite the importance of giving a presentation in professional and educational contexts, there is still no consensus regarding the conceptualization of presentation competence. Instead, approaches and emphases vary across disciplines. Moreover, there are hardly any attempts to combine the theoretical approaches of different disciplines, such as psychology and rhetoric. Based on research in these two fields, a superordinate conceptual framework was deduced that addresses this gap and enables an interdisciplinary and differentiated access to presentation competence which can be transferred to the context of work and organizational psychology. This article concludes with implications for the assessment and demand-oriented promotion of presentation competence.
\end{abstract}

Keywords Presentation competence $\cdot$ Competence framework $\cdot$ Diagnostics $\cdot$ Assessment $\cdot$ Self-evaluation

Evelin Herbein

evelin.herbein@ibbw.kv.bwl.de

Fabian Ruth

fabian.ruth@uni-tuebingen.de

Carmen Lipphardt

carmen.lipphardt@uni-tuebingen.de

Tobias Ringeisen

tobias.ringeisen@hwr-berlin.de
1 Institut für Bildungsanalysen Baden-Württemberg, Stuttgart, Deutschland

2 Hector-Institut für Empirische Bildungsforschung, Eberhard Karls Universität Tübingen, Tübingen, Deutschland

3 Forschungsstelle Präsentationskompetenz am Seminar für Allgemeine Rhetorik, Eberhard Karls Universität Tübingen, Tübingen, Deutschland

4 Berlin School of Economics and Law, Berlin, Deutschland 


\section{Einleitung}

Um beruflich erfolgreich zu sein, wird vor allem von Fachund Führungskräften branchenübergreifend erwartet, gute Präsentationen halten zu können (vgl. Smith und Sodano 2011; van Ginkel et al. 2015a). Dies gilt unter anderem für Berufe in Wirtschaft, Verwaltung, den Naturwissenschaften oder im Gesundheitswesen. Auch Schulabgänger*innen und Hochschulabsolvent*innen weisen der Präsentationskompetenz eine sehr hohe Bedeutung zu, um im Berufsleben Erfolg zu haben (Schaeper und Spangenberg 2008). Präsentationen sind ein kommunikatives Format, um über Sachverhalte zu informieren oder von einer Meinung zu überzeugen (van Ginkel et al. 2015a). Die Kompetenz, die eigene Präsentation an eine Zielgruppe und deren Wissensstand anzupassen und dabei den Kontext zu berücksichtigen, ist demnach bedeutsam, um berufliche Ziele zu erreichen und eigene Ideen erfolgreich zu vertreten (Yurong 2015).

Eine hohe Präsentationskompetenz stellt eine wichtige Anforderung dar, die Unternehmen im Rahmen der Personalauswahl überprüfen. Beispielsweise haben sich Präsentationen als feste Aufgabe in Assessment-Centern etabliert (Hoffman et al. 2015; Krause et al. 2007; Obermann 2018), wobei diese gemäß einer Umfrage von Obermann et al. (2008) in deutschen Unternehmen mit fast $90 \%$ die häufigste Aufgabenkategorie bilden. Zum anderen sind Betriebe daran interessiert, ihre Beschäftigten durch Maßnahmen der Personalentwicklung im Ausbau ihrer Präsentationskompetenz zu unterstützen (z. B. Blickle 2019).

Generell ist das Präsentieren für einen Großteil der Erwachsenen stark mit Angst besetzt (Dwyer und Davidson 2012), was die Bedeutsamkeit erhöht, Beschäftigte mit wenig Präsentationserfahrung wie z.B. Auszubildende und Berufseinsteiger*innen auf diese Aufgabe vorzubereiten. Als Ansatzpunkt liegt mittlerweile eine Vielzahl an Programmen zur Förderung der Präsentationskompetenz vor, deren Wirksamkeit evaluiert wurde (Chan 2012; Lipphardt et al. 2017; Ringeisen und Bürgermeister 2015; Schickel und Ringeisen 2020; van Ginkel et al. 2015a). Die meisten dieser Programme fokussieren auf junge Erwachsene, lassen sich allerdings auf andere Zielgruppen mit abweichendem Erfahrungshintergrund adaptieren, da sich Aufbau und Inhalte mit entsprechenden Förderangeboten im betrieblichen Kontext weitgehend decken (Blickle 2019; Preiser und Turashvili 2016; van Ginkel et al. 2015a). Effektive Präsentationstrainings zu konzipieren, setzt voraus, zu definieren, was unter Präsentationskompetenz verstanden wird und wie diese erfasst werden kann. Unterschiedliche Disziplinen wie die Psychologie, Rhetorik und Sprachdidaktik und -wissenschaft beschäftigen sich mit diesen Fragen. Bisher fehlt jedoch eine disziplinübergreifende Konzeptualisierung von Präsentationskompetenz, welche sich eignet, um Förderangebote insbesondere für Personengruppen mit wenig Präsentationserfahrung wie Jugendliche und junge Erwachsene zu entwickeln (Mulder 2014).

Vor diesem Hintergrund bietet der vorliegende Beitrag einen theoretischen und empirischen Überblick zur Präsentationskompetenz aus multidisziplinärer Perspektive, wobei der Schwerpunkt auf die Psychologie und die Rhetorik gelegt wurde. Dabei wird Bezug genommen zu bestehenden, übergeordneten Kompetenzmodellen, die in der Arbeitsund Organisationspsychologie aber auch der Bildungsforschung verortet sind. Ausgehend von diesem konzeptionellen Rahmen werden verfügbare, validierte Messinstrumente für den deutschsprachigen Raum vorgestellt, die einen multi-methodalen Ansatz der Diagnostik im Rahmen von Personalauswahl und -entwicklung sowie im Bildungskontext eröffnen. Die Mehrzahl dieser Instrumente wurde im Kontext von Trainings zur Förderung von Präsentationskompetenz entwickelt, eignet sich aber auch zum Einsatz bei Fortgeschrittenen mit viel Präsentationserfahrung.

\section{Ausgangspunkt und definitorischer Rahmen}

Bevor Präsentationskompetenz konzeptuell betrachtet wird, fokussieren wir auf das Präsentieren als spezielle Aufgabe und besondere kommunikative Interaktion. Unter Präsentieren verstehen wir disziplinübergreifend das mediengestützte, primär monologische, adressatenorientierte Sprechen, das darauf zielt, eine Zielgruppe zu informieren und/ oder diese zu unterhalten, zu überzeugen bzw. zu emotionalisieren (De Grez et al. 2009; Lobin 2009; Schnettler und Knoblauch 2007). Die Zuhörerschaft besteht dabei mindestens aus einer Person (De Grez und Valcke 2010).

Eine typische Präsentationsaufgabe stellt demnach folgende Interaktionssituation aus der Automobilbranche dar: Ein Entwicklerteam aus Ingenieur*innen, das eine innovative Idee zur Kühlung einer Autobatterie entwickelt hat, stellt diese im Rahmen eines regelmäßig stattfindenden Meetings vor. Bei den Zuhörenden handelt es sich um Vertreter*innen verschiedener Fachabteilungen (z. B. Konstruktion, Elektronik, Softwareentwicklung). Sie sollen anhand einer foliengestützten Präsentation über den neuen Ansatz zur Kühlung informiert und von ihm überzeugt werden, damit dieser in einer neuen Baureihe umgesetzt wird. Ausgehend von diesem Beispiel lassen sich folgende Eigenschaften einer Präsentation ableiten.

Charakteristisch für eine Präsentation ist deren multimodale, multikodale und multimediale Prägung. Multimodalität bezieht sich auf den Umstand, dass eine Präsentation verschiedene kommunikative Modi vereint, wie Informationen auf Folien, die gesprochene Sprache oder Körpersprache (vgl. Bucher et al. 2010). Dies erfordert von Präsentie- 
renden die Verknüpfung visueller, sprachlicher und performativer Informationen in einer kohäsions- und kohärenzstiftenden Weise (Lobin 2009). Bucher (2012) konstatiert, dass in multimodalen Kommunikationskontexten keine Modalität für sich genommen die Bedeutung der Kommunikation erzeugt, sondern diese in deren Zusammenspiel entsteht. Für unser Präsentationsbeispiel bedeutet dies: weder die gesprochenen noch die visualisierten Inhalte der präsentierenden Entwickler*innen schaffen es isoliert voneinander, den Lösungsansatz zur Batteriekühlung verständlich zu machen.

Präsentierende müssen folglich verschiedene Kohäsionsmittel, etwa sprachlicher und visueller Art sowie Mittel modalitätsübergreifender Kohäsionsbildung kennen und diese sinnvoll einsetzen, um einen Zusammenhang herzustellen. Verweishandlungen gehören zu den zentralen Kohäsionsmitteln in Präsentationen (Lobin 2007). Durch diese Handlung, etwa in Form der Gestik, gelingt es der präsentierenden Person, Bezüge zwischen verschiedenen Ebenen herzustellen. So werden zum Beispiel durch gestische Deixis - die Vortragenden zeigen mit einem Finger auf eine Folie - und sprachliche Deixis - sie verknüpfen durch ein mündliches „hier“ ihre Erläuterungen mit dem Inhalt der Folie - die verschiedenen Wirkungsebenen der Präsentation zusammengebracht.

Zusätzlich ist Multikodalität charakteristisch für Präsentationen. Dies bedeutet, dass die von Präsentierenden dargebotenen visuellen, textuellen und performativen Informationen verschiedenen Zeichensystemen entstammen (vgl. Knape 2000). Die gesprochene Sprache gilt ebenso als Zeichensystem wie das ausgewählte visuelle Material (Knape 2000). Das präsentierende Entwicklerteam muss also beispielsweise die Inhalte in sprachliche Kodes (z.B. Englisch) und visuelle Kodes (z.B. schematische Darstellung der Kühlung) übertragen, damit ihre Botschaften möglichst missverständnisfrei dekodiert werden.

Multimedialität bezeichnet den Einsatz verschiedenster Medien in der Präsentation. Rhetorisch betrachtet ist die Beschaffenheit der kommunikativen Mittel von Interesse, die für das effektive rhetorische Handeln beim Präsentieren herangezogen werden (Knape 2000). Präsentierende setzen strategisch Medien ein, wie projizierte Folien, Poster oder andere Objekte. Auch Bild- und Tonträger sowie die körpersprachliche und stimmliche Präsenz des*der Präsentierenden werden als Medium betrachtet, wobei der Auftritt als besonders wichtig erachtet wird (vgl. Knape 2000). Bei der Auswahl müssen die Präsentierenden jedoch berücksichtigen, dass das Medium die Botschaft beeinflusst bzw. deren Übermittlung einschränken oder gar verhindern kann (Knape 2005). Wenn also das Entwicklerteam seinen Ansatz zur Batteriekühlung anhand eines unsauber, von Hand skizzierten Modells präsentieren würde, könnte das dazu führen, dass sowohl die Skizze als auch das Papier als Medium bei den zuhörenden Kolleg*innen den Eindruck erwecken, der Lösungsansatz sei noch wenig ausgereift und undurchdacht. Zusammenfassend machen die Verflechtung verschiedener Modalitäten sowie die Verwendung unterschiedlichster Kodes und Medien die Konzeptualisierung und Diagnostik von Präsentationskompetenz, sowohl als Gegenstand der Forschung als auch der beruflichen Praxis, komplex.

\section{Disziplinäre Perspektiven zur Konzeptualisierung von Präsentationskompetenz}

Verschiedene Disziplinen wie beispielsweise die Sprachwissenschaft, die Rhetorik oder die Psychologie befassen sich mit der Modellierung von Präsentationskompetenz. Dabei verwendet jede Disziplin unterschiedliche Begriffe und Definitionen. Die Sprachwissenschaft versteht unter Präsentieren eine aus verschiedenen Teilprozessen zusammengesetzte Handlungsform, die sie aus linguistischer Perspektive rekonstruieren (vgl. Berkemeier 2006; Gätje et al. 2016). Die Rhetorik betrachtet das Präsentieren als Kommunikationsform aus strategischer Perspektive (vgl. Böhme 2015; Lipphardt 2019; Ruth 2020), während es aus Sicht der Psychologie ein Repertoire an Verhaltensweisen darstellt, deren modulierter Einsatz während einer Präsentation eine hohe Präsentationsleistung ermöglichen (z.B. Bartram 2005).

Obwohl die verschiedenen Disziplinen Präsentationskompetenz unterschiedlich definieren, besteht Einigkeit dahingehend, dass ein*e Sprecher*in dann als kompetent eingeschätzt wird, wenn er*sie effektiv und angemessen präsentiert (vgl. Backlund und Morreale 2015; Herbein et al. 2018; Morreale et al. 2007). Effektiv bedeutet, das Kommunikationsziel der Präsentation zu erreichen, beispielsweise zu informieren. Angemessen meint, dass die Präsentation zum Kontext passt, in dem sie stattfindet. Die Passung bezieht sich dabei auf i) bestehende Normen und Standards, ii) die spezifische Sprechsituation, d.h. den Zeitpunkt, die zur Verfügung stehende Zeit, den Ort sowie den Raum und dessen Ausstattung, iii) das $\mathrm{Pu}$ blikum, iv) das Thema und v) das Kommunikationsziel. Zudem muss vi) die Präsentation zu dem*der Sprecher*in selbst passen (Backlund und Morreale 2015; Herbein 2017; Morreale et al. 2013).

Um einen disziplinübergreifenden konzeptionellen Rahmen zu entwerfen ist es notwendig, die Sichtweisen der einzelnen Disziplinen zusammenzubringen. In einem ersten Schritt werden dazu in diesem Kapitel exemplarisch die Perspektiven von Psychologie und Rhetorik ausführlicher dargestellt, die beide Präsentationskompetenz als ein, sich durch Erfahrung ausdifferenzierendes, situationsangemessenes Präsentationsverhalten verstehen. 


\subsection{Wie lässt sich Präsentationskompetenz in der Psychologie konzeptualisieren?}

Um Kompetenzen zu definieren, bieten sich aus Sicht der Psychologie verschiedene Zugänge an (Kuhn et al. 2016). Eine konzeptuelle Diskussion wird vor allem im Bereich der Pädagogischen Psychologie bzw. der Bildungspsychologie geführt, wobei sich zwei Hauptansätze differenzieren lassen (Blömeke et al. 2015; Kuhn et al. 2016). Einige Forscher*innen vertreten dabei einen analytischen Ansatz (z.B. Weinert 2002), welcher unter Kompetenz die Eigenschaften einer Person versteht, die diese befähigen, sich in einer bestimmten Situation kompetent zu verhalten. Das können kognitive, konative, motivationale und affektive Eigenschaften sein. Andere Wissenschaftler*innen vertreten einen stärker verhaltensorientierten, holistischen Ansatz (z. B. Corno et al. 2002). Dieser definiert Kompetenz als das tatsächliche, effektive Verhalten, das eine Person in einer spezifischen Situation zeigt (Performanz). Im Bereich der Arbeits- und Organisationspsychologie ist der holistische Ansatz vorherrschend (für einen Überblick siehe z.B. Blömeke et al. 2015; Spencer und Spencer 1993). Nach diesem Verständnis bilden Kompetenzen individuelle Merkmale von Personen, die sich in der Güte der resultierenden Verhaltensweisen äußern und somit die Arbeitsleistung in einem gegebenen Kontext und/oder in Bezug auf eine bestimmte Aufgabe determinieren. Ausgangspunkt für die Einschätzung der Präsentationskompetenz einer Person bildet somit das Repertoire der gezeigten Verhaltensweisen in der Präsentationssituation (Bartram 2005; für einen weiteren Überblick zum Kompetenzbegriff siehe Erpenbeck et al. 2017).
Gleichzeitig kann, angelehnt an Blömeke et al. (2015), Präsentationskompetenz als Ressourcenpool verstanden werden, der sich aus verschiedenen Eigenschaften einer Person zusammensetzt, welche wiederum kompetentes Handeln ermöglichen. Diese Eigenschaften beeinflussen beispielsweise, wie die Person eine Präsentation vorbereitet, mit der konkreten Präsentationssituation umgeht und in dieser agiert. Drei kompetenzkonstituierende Dimensionen Wissen, Kompetenzüberzeugungen und Zielorientierungen sowie Verhalten werden dabei sowohl im bildungspsychologischen (De Grez und Valcke 2010; Herbein et al. 2018; van Ginkel et al. 2015a) als auch im organisationspsychologischen Kontext (z. B. Blickle 2019) unterschieden. Diese Dimensionen interagieren und beeinflussen die Vorbereitung sowie das gezeigte Präsentationsverhalten in einer gegebenen Situation. Ferner nehmen Merkmale des Settings auf die Vorbereitung und das Präsentationsverhalten Einfluss (vgl. De Grez und Valcke 2010). Die beschriebenen Prozesse sind in Abb. 1 visualisiert.

Um angemessen und effektiv präsentieren zu können, benötigt eine Person überfachliches Wissen zur Planung und Durchführung von Präsentationen, also über das „Wie“ des Präsentierens (Morreale et al. 2013). Das beinhaltet beispielsweise Wissen zu nonverbaler Kommunikation, d.h. deren Bedeutung und Wirkung, oder Wissen darüber, wie sprachliche Aspekte die Verständlichkeit einer Präsentation erhöhen. Fachliches Wissen über den Redegegenstand repräsentiert hingegen das „Was“ des Präsentierens und wird als Grundvoraussetzung für eine erfolgreiche Präsentation angesehen. Fachliche Expertise steht im oben genannten Beispiel u. a. für ingenieurtechnisches Wissen, welches es den Präsentierenden beispielsweise ermöglicht, passende
Abb. 1 Konzeptioneller Rahmen der Präsentationskompetenz aus psychologischer Perspektive

\section{Konstituierende Merkmale der Präsentationskompetenz}

\begin{tabular}{|c|c|c|}
\hline Eigenschaften der*des Präsentierenden & \multirow[b]{3}{*}{$\begin{array}{l}\text { Vorberei- } \\
\text { tung der } \\
\text { Präsen- } \\
\text { tation }\end{array}$} & \multirow{2}{*}{$\begin{array}{c}\text { Präsentations- } \\
\text { situation }\end{array}$} \\
\hline $\begin{array}{l}\text { Wissen } \\
\text { • zu Planung und Vorbereitung } \\
\text { • zu Durchführung und Präsentationsverhalten }\end{array}$ & & \\
\hline $\begin{array}{l}\text { Kompetenzüberzeugungen \& Zielorientierungen } \\
\text { - Kompetenzüberzeugungen } \\
\text { (z. B. Selbstwirksamkeit) } \\
\text { - Zielorientierungen } \\
\text { (z. B. in Bezug auf Lernen und Leistung) }\end{array}$ & & $\begin{array}{l}\text { Präsentations- } \\
\text { verhalten } \\
\text { Angemessen? } \\
\text { Effektiv? }\end{array}$ \\
\hline $\begin{array}{l}\text { Verhaltenspotenzial } \\
\text { - Nonverbale Kommunikation: visuell und auditiv } \\
\text { - Sprachgebrauch } \\
\text { - Strukturierung } \\
\text { - Visualisierung } \\
\text { - Zeitmanagement }\end{array}$ & $\begin{array}{l}\text { Setting } \\
\text { - Kontext } \\
\text { - Dauer } \\
\text { - Vorbereit } \\
\text { - Zuhörer* }\end{array}$ & mat \\
\hline
\end{tabular}


Visualisierungen zur Batteriekühlung auszuwählen und für Sicherheit beim Präsentieren sorgt.

Als zweites sind die Kompetenzüberzeugungen und Zielorientierungen einer Person bedeutsam. Beispielsweise zeigte sich ein positiver Zusammenhang zwischen dispositionalen Kompetenzüberzeugungen wie Selbstwirksamkeit beim Präsentieren und der gezeigten Leistung in unterschiedlichen Präsentationssettings (De Grez et al. 2009; Ringeisen et al. 2019a, b; Schickel und Ringeisen 2020). Zielorientierungen lassen sich in Annäherungs- und Vermeidungsziele im Hinblick auf die angestrebte Präsentationsleistung und die begleitenden Lernprozesse unterteilen (De Grez et al. 2009). Kompetenzüberzeugungen bilden demnach ab, ob eine Person glaubt, beim Präsentieren angemessenes Verhalten zeigen zu können, während die zugehörigen Zielorientierungen die motivationale Bereitschaft repräsentieren, während einer Präsentation eine gute Leistung zu erzielen bzw. das benötigte Verhaltensrepertoire zu erwerben.

Als drittes benötigen Präsentierende ein Repertoire an Verhaltenspotenzialen, auf das in der Vorbereitungsphase und der Durchführung der Präsentation zurückgegriffen werden kann. Aus theoretischer Sicht lassen sich diese Verhaltenspotenziale verschiedenen Subdimensionen zuordnen (vgl. Ruth et al. 2021; Schreiber et al. 2012). Dazu gehören nonverbal-visuelle (z.B. Blickkontakt, Gestik, Körperhaltung, Mimik) und nonverbal-auditive Verhaltensweisen (z. B. Betonung, Stimme, Sprechgeschwindigkeit), Sprachgebrauch (z.B. Wortwahl, Satzkonstruktion), Strukturierung (z.B. Einleitung, Übergänge), Visualisierung sowie Zeitmanagement. In der Planungsphase sind diese Verhaltenspotenziale beispielsweise bedeutsam, um ein Skript, Visualisierungen und Handouts zu erstellen, die in der Präsentationssituation eingesetzt werden. Das Zusammenspiel der dabei gezeigten Verhaltensweisen muss wiederum zum Setting passen, in dem die Präsentation stattfindet.

Zusätzlich zum Präsentationswissen, den Zielorientierungen und Überzeugungen sowie dem Verhaltenspotenzial wird das gezeigte Präsentationsverhalten von situativ fluktuierenden Variablen wie Bewertungsprozessen - beispielsweise der wahrgenommenen Bedrohung aufgrund einer Zuhörerschaft mit hoher Expertise - und Emotionen - beispielsweise Angst oder Langeweile - begleitet (Schickel et al. 2021; Schickel und Ringeisen 2020). Weder Bewertungsprozesse noch Emotionen bilden einen Teil der Präsentationskompetenz, weshalb diese im Folgenden nicht weiter berücksichtigt werden.

Obwohl sich der analytische und der holistische Ansatz in ihrem Auflösungsgrad unterscheiden, spiegeln beide ein strukturelles Kompetenzverständnis wider. Die vorgestellten Erkenntnisse zur Dimensionalität der Präsentationskompetenz lassen sich somit in einem Kompetenzstrukturmodell zusammenfassen (siehe Abb. 1). Präsentationskompetenz lässt sich wiederum als Facette eines übergeordneten Kompetenzrahmens verorten. Solche Kompetenzrahmen eignen sich, um branchenübergreifend die Bandbreite an Kompetenzanforderungen abzubilden, die als Determinanten der Leistung im beruflichen Kontext betrachtet werden können. In der deutschsprachigen Literatur sind Kompetenzrahmen mit vier übergeordneten Domänen, der Fach-, Methoden-, Sozial- und Selbstkompetenz, sowohl in der Arbeits- und Organisationspsychologie (Schmidt-Rathjens 2007; Sonntag und Schaper 2006; Sonntag und SchmidtRathjens 2005) als auch im Bildungskontext (Hochschulrektorenkonferenz und Kultusministerkonferenz 2017) gängig. In diesen Modellen wird Präsentationskompetenz unter Methodenkompetenz eingereiht. Expertise über den Präsentationsgegenstand hingegen - zum Beispiel ingenieurtechnisches Wissen zur Batteriekühlung - bildet eine Voraussetzung für erfolgreiches Präsentieren und lässt sich der Domäne der Fachkompetenz zuordnen.

Einige Autor*innen schlagen eine differenzierte Struktur vor, die bis zu acht Domänen unterscheidet (Bartram 2005; Schaeper und Spangenberg 2008). In diesen hochauflösenden Kompetenzrahmen lässt sich Präsentationskompetenz theoretisch und empirisch von der Methodenkompetenz abgrenzen und repräsentiert sowohl im beruflichen Kontext (beispielsweise im Modell der Great Eight; Bartram 2005) als auch im Bereich der hochschulischen Bildung (Schaeper und Spangenberg 2008) eine eigene Kompetenzdomäne.

In Bezug auf die Entwicklung von Präsentationskompetenz liegen bisher weder ein empirisch überprüftes Entwicklungsmodell noch ein Niveaumodell vor, welche die Veränderungsprozesse bzw. die Entwicklungsstufen der Präsentationskompetenz abbilden. Basierend auf ersten Hinweisen besteht allerdings die Annahme, dass sich die Fähigkeit, angemessen und effektiv präsentieren zu können, schrittweise entwickelt (vgl. Herbein 2017; Rubin und Morreale 1996; Staton und Tomlinson 2001). Dabei lassen sich ein basales und ein fortgeschrittenes Level unterscheiden. Auf dem basalen Level haben die Präsentierenden bereits bestimmte Verhaltensweisen erlernt, auf welche sie in Präsentationssituationen zurückgreifen können. Die Wahl und der praktische Einsatz sind dabei noch nicht spezifisch angepasst an die jeweilige Präsentationssituation. Erst auf fortgeschrittenem Level sind die Präsentierenden in der Lage, aus dem erworbenen Repertoire passende Verhaltensweisen so auszuwählen und anzupassen, dass diese angemessen für die Präsentationssituation sind. Die vorgestellten Überlegungen zu abgrenzbaren Niveaustufen der Präsentationskompetenz spielen in der Förderpraxis, beispielsweise im schulischen Deutschunterricht, bereits eine wichtige Rolle, auch wenn ein empirischer Gültigkeitsnachweis noch aussteht (Geldmacher 2010). 


\subsection{Wie lässt sich Präsentationskompetenz rhetorisch konzeptualisieren?}

Auch in der Rhetoriktheorie ist ein holistischer Ansatz der Kompetenz angelegt, da das Präsentieren als rhetorische Handlung nur vor dem Hintergrund der Präsentationssituation versteh- und bewertbar ist (vgl. Kramer und Gottschling 2012). Hinsichtlich einer konkreten Präsentationssituation sind für die Rhetorik Faktoren wie z.B. Adressat, Thema und Kontext bedeutsam, weil sie den Handlungsspielraum der*des Präsentierenden definieren. Rhetorisch betrachtet ist auch die Planungsphase bedeutsam, denn die Antizipation der Präsentationsituation ermöglicht der*dem Präsentierenden die bestmögliche Vorbereitung und die treffende Auswahl aus der Vielzahl rhetorischer Vorgehensweisen. Sie ist grundlegend für eine strategische, an einem bestimmten Ziel ausgerichtete Präsentation. Als charakteristisches Spezifikum der Rhetorik gilt die strategische Ausrichtung der Kommunikation am Publikum (vgl. z. B. Aristoteles 2002, I, 3, 1358b; Kramer und Gottschling 2012; Ueding und Steinbrink 2011). Damit stellt die Situationsund Adressatenanalyse den zentralen Ausgangspunkt bei der konkreten Präsentationsvorbereitung dar (Kramer und Malaka 2014). Für die Präsentierenden aus unserem Beispiel ist es demnach notwendig, die technische Ausstattung des Meetingraums zu bedenken und das Vorwissen der anwesenden anderen Teams (Konstruktion, Elektronik, Softwareabteilung) zu Kühlungsprozessen oder vorhandene Stimmungen in Erfahrung zu bringen. Davon lassen sich dann geeignete Kommunikationsstrategien ableiten. Kompetentes rhetorisches Verhalten erfordert von der*dem Präsentierenden, geeignete Kommunikationsmittel zu identifizieren und auszuwählen sowie diese dann so auszugestalten, dass erfolgreiches Präsentieren in einer gegebenen Situation resultiert (vgl. Knape 2013).

In der Rhetoriktheorie lassen sich hinsichtlich der Analyse des kompetenten Präsentierens zudem die Phasen der Vorbereitung sowie die Phase der eigentlichen Präsentation (actio) unterscheiden, die sich aus den sechs Produktionsstadien der klassischen Rede ableiten. Diese umfassen intellectio (die Analyse des kommunikativen Settings) sowie inventio, dispositio, elocutio, memoria und actio, wobei die letztgenannten fünf Bereiche die Elemente Sammeln von Gedanken zum Redegegenstand, Strukturieren der Gedanken, Formulieren, Einprägen ins Gedächtnis und die tatsächliche Aufführung der Präsentation darstellen. Die Produktionsstadien greifen jeweils ineinander und bedingen sich gegenseitig (Ueding und Steinbrink 2011). Alle Arbeitsstadien werden in der Rhetorik ausführlich behandelt. So hat sich beispielsweise im Bereich der Sprachgestaltung (elocutio) eine detaillierte Lehre zu Stiltugenden (u.a. zur Angemessenheit), zum Redeschmuck (rhetorische Figuren), den Wirkungsfunktionen oder auch zur Ausgestaltung der verschiedenen Redeteile herausgebildet. Beispielsweise ist das Wissen um die rhetorische Figur des Paradoxons für die Vortragenden in unserem Beispiel hilfreich, um einen aufmerksamkeitserregenden Einstieg zu generieren und die paradoxe Lösungsidee der Batteriekühlung herauszuarbeiten (wir kühlen mit Hitze). Moderne Ansätze der Rhetoriktheorie erweitern diese Lehren etwa um die Aspekte der medialen Bedingungen (vgl. Knape 2000). Die so entstandene rhetorische Systematik ermöglicht die Ausbildung der Präsentationskompetenz auf der Wissensebene und die Spezifizierung der Verhaltensebene. Die dritte Dimension der Zielorientierung der*des Präsentierenden stellt den Kern der Rhetoriktheorie dar. Zur Zielerreichung gehört die eigene Inszenierung der präsentierenden Person, wenn etwa die eigene Fachkompetenz in der Präsentation aufgezeigt wird. Zusätzlich sind logos (das rationale Argument) und pathos (das Publikum in eine bestimmte Gefühlslage zu versetzen) Überzeugungsmittel der Rede.

Der psychologischen Perspektive vergleichbar werden in der Rhetorik kompetenz-konstituierende Dimensionen unter anderem in der exercitatio (Übung) verortet, die der Präsentationsvorbereitung zuzuordnen ist. Rhetorische Kompetenzförderung lässt sich durch die Fokussierung auf einzelne Produktionsstadien, z.B. sprachliche Ausdrucksformen finden (etwa sprachliche Anschaulichkeit erzeugen) oder Strukturieren (etwa aufmerksamkeitsstiftende Einleitungen gestalten), verwirklichen. Sie lässt sich ebenso mit Übungssituationen entwickeln, die alle Produktionsstadien gleichzeitig ansprechen und das Präsentationshandeln simulieren, aber noch nicht die reale Präsentationssituation darstellen. Eine anschauliche und nachvollziehbare Erklärweise der Präsentierenden, etwa technische Zusammenhänge der Batterieentwicklung mit Hilfe von Vergleichen oder Beispielen aus dem Alltagsleben darzustellen, leistet einen entscheidenden Beitrag zum gemeinsamen Verständnis der Kommunikationspartner*innen und erleichtert schließlich den fachlichen Austausch verschiedener Expert*innen. Passende Vergleiche oder Beispiele auszuwählen, erfordert von Präsentierenden nicht nur Fachwissen (z.B. zur Batteriekühlung), sondern zudem ein Bewusstsein für die Herstellung des Adressatenbezugs (Vergleiche funktionieren nur, wenn der Vergleichsgegenstand bekannt ist) sowie grundlegendes Wissen zum Potenzial der Sprache. Dies zeigt, dass neben anderen Bereichen auch die sprachliche Gestaltung bei der Förderung der Präsentationskompetenz in den Blick genommen werden muss.

Aus rhetorischer Perspektive sind folglich für die Diagnostik von Präsentationskompetenz die gehaltene Präsentation sowie die Produkte innerhalb der Produktionsstadien von Interesse. Insgesamt bildet die rhetorische Systematik die Grundlage für die Vorbereitung der Präsentationssituation. Für die konkrete Ableitung von Handlungsmustern (Vorbereitung der Präsentation) ist der Transfer aus 
Abb. 2 Konzeptioneller Rahmen der Präsentationskompetenz aus rhetorischer Perspektive

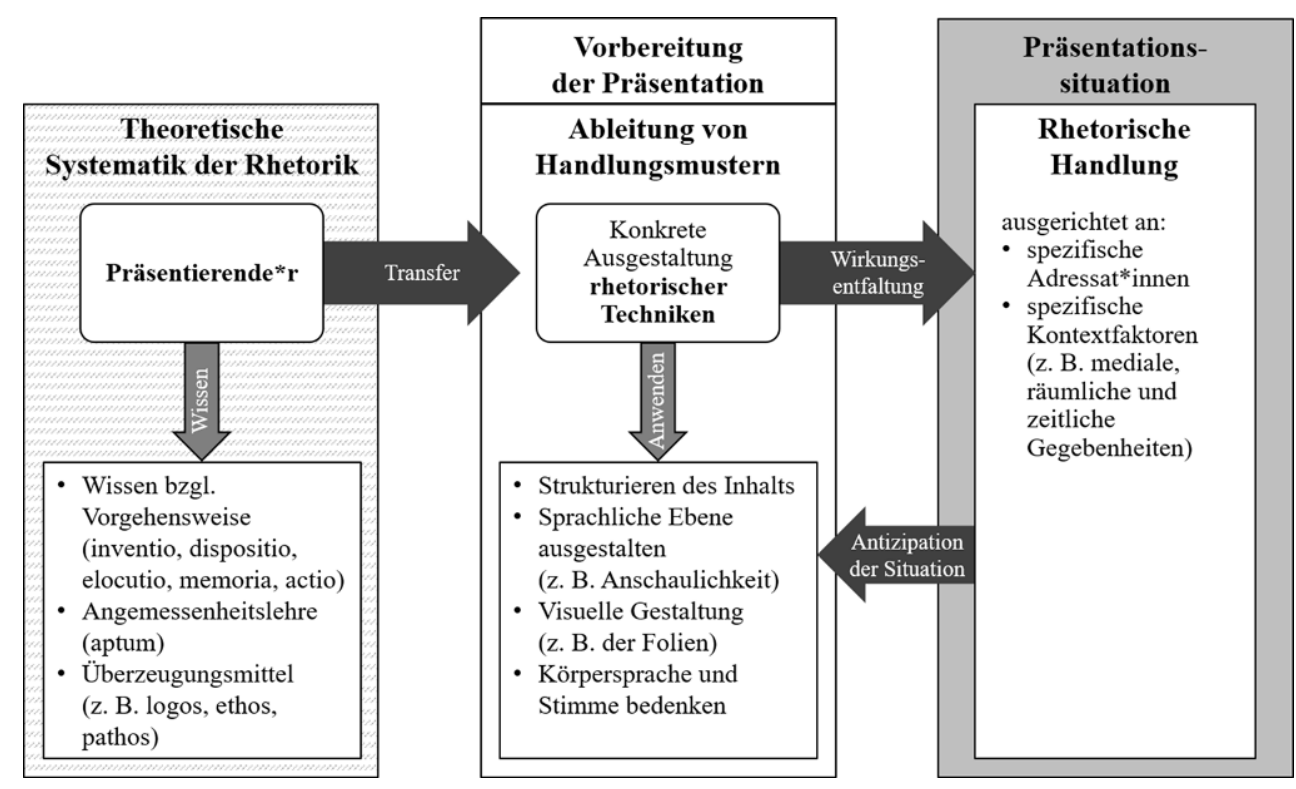

der rhetorischen Theorie sowie die Antizipation der Präsentationssituation ausschlaggebend. In Abb. 2 zeigen wir den Handlungsablauf von der theoretischen Systematik der Rhetorik bis zur rhetorischen Handlung in der konkreten Präsentationssituation auf.

Vor dem Hintergrund einer disziplinübergreifenden Betrachtung von Präsentationskompetenz weist die rhetorische Perspektive zahlreiche Gemeinsamkeiten zum psychologischen Rahmenkonzept auf. Dazu zählt das holistische Verständnis der Präsentationskompetenz mit Fokus auf dem zielgerichteten und effektiven Präsentationsverhalten sowie die Betonung der Angemessenheit des Verhaltens in der Präsentationssituation. Damit fällt auch aus rhetorischer Perspektive dem konkreten Handeln in der Präsentationssituation eine besondere Wichtigkeit zu. Zugleich werden jedoch innerhalb der Rhetorik die verschiedenen Dimensionen anders zusammengefasst und subsumiert; so ist Kompetenzüberzeugung kein genuin rhetorischer Begriff, doch spielen Teilaspekte davon in der Rhetorik eine Rolle, wie zum Beispiel die Inszenierung der Rednerpersönlichkeit. Dennoch bietet die interdisziplinäre Betrachtung von Präsentationskompetenz die Möglichkeit, einander theoretisch, zum Beispiel bei der Ableitung eines Entwicklungsmodells der Präsentationskompetenz, und methodisch, zum Beispiel bei der Entwicklung eines Wissenstests, zu ergänzen. So kann bei der Entwicklung von Items auf die umfassende Rhetoriktheorie zu sprachlichen Mitteln zurückgegriffen werden.

\section{Diagnostik der Präsentationskompetenz und deren konstituierender Merkmale}

Die Entwicklung von Messinstrumenten für die Erfassung der Präsentationskompetenz ist abhängig von deren Modellierung. Gemäß des analytischen Ansatzes werden die zugrundeliegenden Eigenschaften einer Person, die das gezeigte Präsentationsverhalten beeinflussen (Wissen, motivationale und somit handlungsleitende Kompetenzüberzeugungen und Zielorientierungen sowie Verhaltenspotenziale), erfasst. Im Rahmen des holistischen Ansatzes wird das Verhalten in der Präsentationssituation eingeschätzt und dabei beurteilt, inwieweit die Präsentierenden effektiv und angemessen handeln (vgl. De Grez et al. 2009; Herbein et al. 2018; van Ginkel et al. 2015a). Um den Gegenstand der Präsentationskompetenz je nach Ansatz diagnostisch zu erfassen, stehen validierte Selbst- und Fremdberichtsverfahren zur Verfügung.

Um einen systematischen Überblick über Instrumente zur Erfassung der Präsentationskompetenz im Bereich der Arbeits- und Organisationspsychologie zu erhalten, wurde eine umfassende Literaturrecherche durchgeführt. Diese zeigte, dass kaum validierte Instrumente vorliegen zur Erfassung von a) der Angemessenheit des gezeigten Präsentationsverhaltens, welches sich, wie zuvor vorgestellt, in theoretisch abgrenzbare Facetten unterteilen lässt, oder b) den zugrundeliegenden Eigenschaften einer Person, die das gezeigte Präsentationsverhalten beeinflussen. In der Forschung und Praxis der Pädagogischen Psychologie und der Rhetorik liegen hingegen validierte Instrumente vor, deren psychometrische Eigenschaften überprüft wurden, um die vorgestellten Facetten und konstituierenden Merkmale der Präsentationskompetenz zuverlässig zu erfassen. 
Daher fokussiert dieser Beitrag im Folgenden zunächst auf letztgenannte Instrumente, bevor im Anschluss auf die Diagnostik von Präsentationskompetenz in der organisationspsychologischen Praxis eingegangen wird.

\subsection{Erfassung des Wissens zum Präsentieren}

Präsentationsbezogenes Wissen stellt eine wichtige reflexive Ebene des Präsentationshandelns dar, um das eigene kommunikative Ziel zu erreichen (vgl. Knape 2013), sowohl für die Vorbereitung als auch die Präsentationssituation. Nur wenige Studien verweisen auf einen Wissensfragebogen mit Bezug zur Präsentationskompetenz (z. B. Boser et al. 2014). Allerdings gibt es dazu keine näheren Ausführungen.

Bislang ist die Wissensebene und im Speziellen die Wissensebene zur Erzeugung von Anschaulichkeit mittels rhetorischer Techniken in der Forschung wenig repräsentiert, obwohl gerade die sprachliche Gestaltung für die fachübergreifende Wissenskommunikation bedeutsam ist. Im Rahmen einer Studie mit Jugendlichen wurde hierfür ein neuer Test entwickelt. Dieser erfasst präsentationsbezogenes Wissen, insbesondere über solche rhetorische Techniken, die beim Publikum während der Präsentation Anschaulichkeit erzeugen können (Lipphardt 2019). Anschaulichkeit ist vor allem in wissensvermittelnden Präsentationen bedeutend, um - wie bereits weiter oben skizziert wurde - durch sie komplexe Sachverhalte für das Publikum verständlicher zu machen. Rhetoriktheoretisch geht es dabei darum, sprachlich bei den Adressat*innen Vorstellungen und Einsichten vom behandelten Redegegenstand zu erzeugen, so dass dieser verständlich wird. Bezogen auf das Präsentationsbeispiel zu einem neuen Ansatz der Batteriekühlung bedeutet dies, den Kolleg*innen anderer Fachabteilungen, denen bestimmtes Fachwissen fehlt, die Inhalte so zugänglich zu machen, dass sie trotzdem in die Lage versetzt werden, sich ein eigenes Urteil zu bilden. Sprachliche Mittel wie Vergleiche können ausgehend vom Fachwissen der Adressat*innen somit eine Brücke zum neuen Sachverhalt bauen.

Mit der Messung von präsentationsbezogenem Wissen sind verschiedene Problemstellungen verbunden. Hierzu gehört, dass kommunikatives Handeln in einer Präsentation letztendlich kontextgebunden ist; es damit schwierig ist zu beurteilen, mit welchen konkreten Effekten eine sprachliche Technik verbunden ist. Der Test erfasst Wissen zur Anschaulichkeitserzeugung mittels rhetorischer Techniken, etwa in Bezug auf die sprachliche und visuelle bzw. mediale Gestaltung der Präsentation, wobei der Schwerpunkt auf den sprachlichen Techniken liegt, die im Zusammenhang mit Lernen, etwa in verschiedenen Fachdidaktiken diskutiert werden (vgl. z.B. Brünner 2013; Wyßuwa und Beier 2013; Kliche 2013). Sprachliche Techniken, wie etwa Vergleiche, sind vor allem in der Experten-Laien-Kommu- nikation vorstellungs- und verständnisfördernd, was sich somit im Beispiel auch positiv auf die präsentationsgestützte, abteilungsübergreifende Kommunikation über die Batteriekühlung zwischen dem Entwicklerteam und den fachfremden Kolleg*innen auswirken sollte.

Der Test gliedert sich in sechs Subskalen: 1) Beispiel, 2) Vergleich, 3) Detaillierung, 4) visuelle bzw. mediale Gestaltung, 5) Struktur, 6) Verständlichkeit. Alle Bereiche sind durch jeweils vier Items abgedeckt, zusätzlich sind Distraktoren integriert. Die Items sind als Aussagesätze formuliert und beziehen sich jeweils auf einen der oben genannten Bereiche (Beispielitem aus der Subskala „Vergleich“: „,Mit Vergleichen lässt sich Neues aus Bekanntem nicht erschließen."). Zur Einschätzung der Items wird eine vierstufige Skala genutzt $(1=$ stimmt gar nicht bis $4=$ stimmt genau $)$. Die Konzeption des Tests erfolgte theoriegeleitet und basiert auf der rhetorischen elocutio-Lehre (vgl. z. B. Ueding und Steinbrink 2011; Lipphardt 2019). Psychometrische Angaben zu diesem Test können noch nicht berichtet werden, da die Entwicklung noch nicht abgeschlossen ist.

\subsection{Erfassung der Kompetenzüberzeugungen und Zielorientierungen zum Präsentieren}

Im Bereich der Kompetenzüberzeugungen bildet die Selbstwirksamkeit beim Präsentieren eine personenbezogene Ressource, die es der*dem Präsentierenden erleichtert, während einer Präsentation angemessenes Verhalten zu zeigen. Zur Erfassung im Selbstbericht eignet sich eine Skala mit 8 Items von Ringeisen et al. (2019b), die Kompetenzüberzeugungen bezüglich verbaler und nonverbaler Fertigkeiten beim Präsentieren erfasst (z. B. „Kommen während einer Präsentation unerwartete Fragen, so kann ich diese jederzeit beantworten."). Zur Einschätzung kann eine vier- oder fünfstufige Skala genutzt werden $(1=$ trifft gar nicht $z u$ bis 4 bzw. $5=$ trifft voll $z u$ ). Gute psychometrische Kennwerte mit Cronbach's Alpha >0,80 und multiple Hinweise zur Faktor-, Konstrukt- und Kriteriumsvalidität liegen vor (Ringeisen und Bürgermeister 2015; Ringeisen et al. 2019a, b; Schickel et al. 2021; Schickel und Ringeisen 2020). Während einer Präsentation begünstigt Selbstwirksamkeit beispielsweise den verhaltensbezogenen Aufbau von Präsentationskompetenz, ermöglicht ein angemessenes nonverbales und verbales Präsentationsverhalten und mildert leistungshinderliche Stressreaktionen wie Angsterleben oder erhöhte Kortisolausschüttung ab. Im Rahmen von Präsentationstrainings lässt sich der Aufbau von präsentationsbezogener Selbstwirksamkeit fördern, welche wiederum lernhinderliche Emotionen wie Langeweile reduziert und somit direkt und indirekt zu einer besseren Präsentationsleistung beiträgt.

Ferner stehen Selbstberichtinstrumente zur Verfügung, um Zielorientierungen im Sinne von Annäherung und Ver- 
meidung in Bezug auf Leistung und Lernprozesse beim Präsentieren zu erfassen (für einen Überblick siehe z.B. De Grez et al. 2009; van Ginkel et al. 2015a, b). Bei der Messung hat sich bewährt, kontextunspezifische Items einzusetzen und den Bezug zum Präsentieren über eine einleitende Formulierung herzustellen (z. B. „Beim Erwerb von Präsentationskompetenzen ..."). Gut eignet sich die deutsche Version (Bachmann 2009) des Revised Academic Goal Questionnaire (AGQ-R; Elliot und Murayama 2008), welche die vier Facetten Lernannäherung, Lernvermeidung (z.B. „,... mache ich mir Sorgen, dass ich nicht alles lerne, was ich lernen könnte.“), Leistungsannäherung (z. B. ,... ist es für mich wichtig, besser zu sein als andere Lernende.") und Leistungsvermeidung jeweils über 3 Items erfasst. Obwohl bisher keine Validierungsstudien vorliegen, wurde die Dimensionalität des deutschen AGQ-R im Rahmen von Projekten zur Förderung von Präsentationskompetenz konfirmatorisch überprüft und erbrachte Nachweise der Faktorund Konstruktvalidität sowie gute psychometrische Kennwerte mit Cronbach's Alpha $\geq 0,80$ für alle Subskalen (z. B. Ringeisen und Bürgermeister 2015; Ringeisen et al. 2019b; Schickel und Ringeisen 2020).

\subsection{Erfassung der Angemessenheit des gezeigten Präsentationsverhaltens}

Zur Einschätzung des (non)verbalen Präsentationsverhaltens bei jungen Erwachsenen liegen verschiedene verhaltensbasierte Fremdbeurteilungsbögen vor (für einen Überblick siehe z. B. De Grez et al. 2009; Schreiber et al. 2012; van Ginkel et al. 2015b). Fremdbeurteilungsbögen eignen sich, um die gezeigten verbalen und nonverbalen Verhaltensweisen beim Präsentieren durch Beobachter*innen präzise und zeitökonomisch einzuschätzen und Feedback im Rahmen von Präsentationstrainings zu geben. Die Bögen wurden theoriegeleitet entwickelt und erfassen die interessierenden Verhaltensfacetten im Sinne einer Beobachtungsskala im Allgemeinen anhand eines Items. Das erwünschte Verhalten wird dabei explizit beschrieben und entweder anhand einer mehrstufigen oder binären Ratingskala nach Häufigkeit (z.B. von fast nie bis fast immer) oder Intensität (z.B. von trifft gar nicht $z u$ bis trifft voll zu) von einem oder mehreren Rater*innen eingeschätzt. Bei Bedarf lassen sich Raterübereinstimmungen berechnen. Als Beispiel findet sich in Anhang 1 ein Fremdbeurteilungsbogen, der in Anlehnung an De Grez et al. (2009) von Ringeisen und Kolleg*innen entwickelt wurde und für den multiple Nachweise einer adäquaten Konstrukt- und Kriteriumsvalidität vorliegen (z.B. Ringeisen und Bürgermeister 2015; Ringeisen et al. 2019b; Schickel und Ringeisen 2020). Weitere deutschsprachige Fremdbeurteilungsbögen liegen vor, zu denen die Autor*innen allerdings keine Validitätsangaben liefern (z.B. Boser et al. 2014; Treutner 2016).
Weiterhin liegt ein validierter Fremdbeurteilungsbogen vor, der die interessierenden Facetten des Präsentationsverhaltens jeweils anhand einer Skala mit mehreren Items erfasst. Es handelt sich um das Tübinger Instrument für Präsentationskompetenz (TIP), das für die Erfassung des Präsentationsverhaltens von Sekundarstufenschüler*innen entwickelt und hinsichtlich seiner Objektivität, Reliabilität und Validität überprüft wurde (Ruth et al. 2021). Die Konzeption des Instruments basierte auf dem holistischen Ansatz der Präsentationskompetenz. Sechs Facetten des Präsentationsverhaltens werden erfasst. Diese wurden aus der Rhetoriktheorie abgeleitet. Dabei bildeten die Produktionsstufen einer klassischen Rede, welche um die Erfordernisse des Präsentationsformates ergänzt wurden, die Grundlage. Die Facetten sind 1) Adressatenorientierung, 2) Struktur, 3) Sprachgebrauch, 4) Körpersprache und Stimme, 5) Medieneinsatz und 6) sachliche Glaubwürdigkeit. Das TIP besteht aus 22 Items (z.B. „Der Präsentierende spricht das Publikum an.“), welche auf einer vierstufigen Skala beantwortet werden ( $1=$ trifft gar nicht $z u$ bis $4=$ trifft voll $z u)$. Ausgangspunkt der Einschätzungen sind gefilmte, dreiminütige Schülerpräsentationen. Anhand eines Manuals - mit Ankerbeispielen für jedes Item - und einer Schulung mit Beispielvideos wurden externe Rater*innen in der Anwendung des Instrumentes geschult.

Bei der psychometrischen Überprüfung des TIP $\left(N_{\text {Videos }}=254\right)$ zeigte sich eine zufriedenstellende Interrater-Reliabilität (Objektivität) - bei 15 von 22 Items lagen Intraklassen-Korrelationskoeffizienten von über 0,60 vor sowie erste Hinweise für eine zufriedenstellende RetestReliabilität (die Korrelationen liegen im Bereich zwischen 0,25 und 0,73 bei den Facetten). In einer anschließenden explorativen Faktorenanalyse wurden nur Items mit einer ausreichend hohen Intraklassenkorrelation (ICC) von über 0,60 aufgenommen. Zudem wurden Items nur dann berücksichtigt, wenn insgesamt mindestens drei Items pro theoretisch angenommener Facette aufgrund ausreichender ICC eingeschlossen werden konnten. In der explorativen Faktorenanalyse konnte eine dreidimensionale Struktur ermittelt werden, die den theoretisch abgeleiteten Facetten Adressatenorientierung, Körpersprache und Stimme sowie Medieneinsatz entsprach. Zur weiteren Überprüfung der Validität wurde das mittels des TIP erfasste Präsentationsverhalten mit weiteren präsentationskompetenznahen und -fernen Konstrukten korreliert. Moderate bis hohe Korrelationen fanden sich zwischen dem TIP und Expert*innen-Liveratings der Präsentationen (zwischen 0,43 und 0,67); geringe und moderate Korrelationen fanden sich mit dem selbsteingeschätzten Präsentationsverhalten der Schüler*innen (zwischen 0,13 und 0,31). Diese Ergebnisse sind konform mit bisherigen Forschungsbefunden. Außerdem konnten die erwartungskonformen, negativen Zusammenhänge des TIP mit Redeängstlichkeit (zwischen 
$-0,20$ und $-0,45)$ die Validität stützen. Die Befunde sprechen dafür, das TIP als Ratinginstrument in weiteren wissenschaftlichen Studien, wie z.B. Effektivitätsstudien, und in pädagogischen Kontexten als ergänzende Rückmeldung von externen Expert*innen einzusetzen. Gleichzeitig zeigen die Ergebnisse Weiterentwicklungsbereiche für das Instrument auf.

Neben den vorgestellten Fremdbeurteilungsverfahren stehen auch zwei validierte Instrumente zur Verfügung, um Präsentationskompetenz im Sinne effektiven Präsentationsverhaltens durch Selbsteinschätzung zu erfassen. Das Berliner Evaluationsinstrument für selbsteingeschätzte, studentische Kompetenzen (BEvaKomp; Braun et al. 2008) erfasst die Veränderung des Kompetenzniveaus in verschiedenen Kompetenzdomänen, wobei Präsentationskompetenz anhand von 3 Items erfasst wird. Da aufgrund der geringen Itemzahl nur ein Ausschnitt des Präsentationsverhaltens erfasst werden kann, empfiehlt es sich zur Verbesserung der Konstruktvalidität, Items zu ergänzen, um zusätzliche Verhaltensfacetten beim Präsentieren abzubilden. Dazu eignen sich Items aus validierten Instrumenten, die multiple Facetten des Präsentationsverhalten abbilden, beispielsweise das TIP (Ruth et al. 2021) oder die Skala zur Erfassung der Selbstwirksamkeit beim Präsentieren (Ringeisen et al. 2019b). Bisherige Studien zum BEvaKomp berichten gute psychometrische Kennwerte und multiple Hinweise zur Faktor-, Konstrukt- und Kriteriumsvalidität, sowohl für die Originalskala „Präsentationskompetenz“ (z. B. $\alpha>0,80$; Braun et al. 2008; Braun und Hannover 2011) als auch eine erweiterte Skala mit 6 Items (z.B. $\alpha>0,95$; Schickel und Ringeisen 2020).

Der Fragebogen zur Selbsteinschätzung von Trainerkompetenzen (SKT; Buchholz und Eberle 2003) erfasst anhand von jeweils 4 Einzelitems insgesamt acht Kompetenzbereiche, über die Dozent*innen verfügen sollten, die sozialkommunikativ-methodische Kompetenzen vermitteln. Die zwei Kompetenzbereiche „Selbstsicheres Auftreten“ und „Informationen vermitteln“ lassen sich als Teilbereiche von Präsentationskompetenz konzeptualisieren. Der SKT wurde in einer Vielzahl an Studien zur Kompetenzförderung in beruflichen und hochschulischen Settings eingesetzt, die zusammenfassend eine gute faktorielle Validität (explorative und konfirmatorische Prüfung), gute Kriteriumsvalidität (Korrelationen zum Umgang mit kritischen Situationen), jedoch nur mäßige bis gute Reliabilitätswerte aufwiesen $(\omega=0,57$ bis 0,87; Boser et al. 2014; Buchholz 2009; Herdel und Preiser 2018; Preiser und Turashvili 2016).

\subsection{Diagnostik von Präsentationskompetenz in der organisationspsychologischen Praxis}

Präsentationskompetenz bildet in vielen Berufen einen Teil des Anforderungsportfolios, das Bewerber*innen erfüllen müssen, um eine vakante Position zu erhalten (Obermann et al. 2008; Schaeper und Spangenberg 2008). In der Arbeits- und Organisationspsychologie kommt der Erfassung von Präsentationskompetenz somit im Rahmen der Personalauswahl und der Personalentwicklung eine hohe Relevanz zu. In der Personalauswahl ist ein strukturelles Kompetenzverständnis vorherrschend, welches vorrangig die Perspektive der Arbeitgeber*innen abbildet (Schuler 2006). Die Spezifikation der Anforderungen erfolgt im Allgemeinen anhand übergeordneter Kompetenzrahmen, die branchen- und tätigkeitsübergreifend die Bandbreite berufsrelevanter Kompetenzen abbilden. Somit liegen theoretisch verortete Publikationen zu ausgefeilten Strukturmodellen vor, die Präsentationskompetenz als Teil eines übergeordneten Kompetenzrahmens konzeptualisieren, dabei aber kaum deren konstituierende Teilfacetten differenzieren (z.B. Bartram 2005; Schmidt-Rathjens 2007; Sonntag und SchmidtRathjens 2005).

Im Rahmen von Personalauswahlverfahren wird Präsentationskompetenz somit selten als eigenes Konstrukt, sondern zusammen mit anderen Kompetenzanforderungen erfasst. Die Personalauswahl interessiert sich weniger für eine hochauflösende Erfassung der Facetten von Präsentationskompetenz, sondern für eine umfassende aber ökonomische Erfassung aller für eine Tätigkeit relevanter Kompetenzanforderungen. Entsprechend dieser Ausrichtung fokussiert die Personalauswahl vorrangig auf Fremdberichtsverfahren, mit deren Hilfe sich die Kompetenzprofile von Bewerber*innen, beispielsweise im Rahmen eines AssessmentCenters, schnell einschätzen und vergleichen lassen (für einen Überblick siehe z.B. John 2007; Obermann 2018). Instrumente, die die Kompetenzen und deren Veränderung im Selbstbericht erfassen, finden in der Personalauswahl kaum Anwendung.

Im Sinne eines Multi-Trait-Multi-Method-Ansatzes dienen Präsentationsaufgaben in Assessment-Centern dazu, eine soziale Situation zu inszenieren, anhand derer multiple Kompetenzanforderungen und Persönlichkeitseigenschaften erhoben werden (Freimuth und Sieland 2013; Obermann 2018; Rahn und Moser 2000). Werden Instrumente zur Erfassung der Präsentationskompetenz oder mit ihr verwandter Konstrukte eingesetzt, dann erfolgt die Einschätzung meist auf einem globalen Niveau (vgl. Preiser und Turashvili 2016). Eine detaillierte Erfassung der Präsentationsverhaltensweisen, die den theoretisch abgrenzbaren Facetten der Präsentationskompetenz zugeordneten werden können, sowie die zugrundeliegenden Eigenschaften einer Person, die das gezeigte Präsentationsverhalten beeinflussen, erfolgt kaum.

Es liegen übergeordnete Gestaltungshinweise für Präsentationsaufgaben im Rahmen von Assessment-Centern sowie erste Nachweise ihrer Validität vor (Hoffman et al. 2015). Allerdings lassen sich nach Schuler (2006) die Prä- 
sentationsaufgaben in Assessment-Centern kaum vergleichen, da die Kompetenzanforderungen der zu besetzenden Stelle die Art und den Gegenstand der Präsentationsaufgabe bestimmen. Als Folge variieren die Verhaltensweisen, die von den Vortragenden erwartet und von den Beobachter*innen beurteilt werden. Zudem wird neben (oder anstelle) des reinen Präsentationsverhaltens oft auch der Inhalt der Präsentation (wenn z.B. Fachwissen evaluiert werden soll) oder die erzielte Wirkung der*des Vortragenden bewertet (Freimuth und Sieland 2013; Obermann 2018; Schuler 2006). Vor diesem Hintergrund sprechen die genannten Autor*innen Empfehlungen aus, welche Charakteristika Präsentationsaufgaben in einem Assessment-Center aufweisen sollten und unter welchen Bedingungen diese einzusetzen und durchzuführen sind. Beispielsweise wird empfohlen, für die interessierenden Kompetenzfacetten einfache Verhaltensanker zu formulieren, deren Häufigkeit/Intensität dann bewertet wird (für ein Beispiel siehe Freimuth und Sieland 2013, S. 673). Ein solches Vorgehen erschwert den Einsatz differenzierter, theoriegeleiteter, validierter und standardisierter Beobachtungsskalen. Um die Genauigkeit der Einschätzung durch die Auswahlkommission zu erhöhen und Beurteilungsfehler wie den Halo-Effekt oder Verzerrungen aufgrund von Attraktivität oder Sympathie zu reduzieren, werden ausführliche Trainings der Beobachtenden empfohlen (Borman et al. 2010). Unter Berücksichtigung der vorgestellten Gestaltungsprinzipien kommen Rahn und Moser (2000) zum Schluss, dass für den deutschsprachigen Kontext bisher kaum Evaluationsstudien zur Reliabilität und Validität einer Präsentationsübung im Rahmen eines Assessment-Centers vorliegen.

Im Rahmen der Personalentwicklung geht es um die Förderung berufsrelevanter Kompetenzen, wobei Präsentationskompetenz mit angemessenem Präsentationsverhalten gleichgesetzt wird, das sich mit Hilfe von Trainings verbessern lässt (Blickle 2019; Sonntag und Schaper 2006). Die Personalentwicklung nimmt somit eine entwicklungsorientierte Perspektive auf Präsentationskompetenz ein. Dabei ist es von Interesse, die Verhaltensweisen beim Präsentieren einfach, zeitökonomisch und individualisiert zu erfassen und diese durch Feedback gezielt zu verbessern. Präsentationstrainings gehören zum Standardrepertoire des Angebotsportfolios in der Personalentwicklung, wobei eine hohe Variabilität an Trainingskonzepten existiert, die sich u. a. an den Kundenwünschen und den Bedürfnissen der Teilnehmer*innen orientieren. Für ausgewählte interaktionsintensive Berufsgruppen wie Hochschullehrende, Lehrer*innen oder Berater*innen wurde die Wirksamkeit kommunikationsorientierter Maßnahmen wie Präsentationstrainings anhand validierter Fremdratingverfahren evaluiert, wobei ausgewählte Verhaltensfacetten wie „Klarheit der Präsentation“ und „Interessantheit der Präsentation“ eingeschätzt wurden, die sich als Teilaspekte der Präsentationskompetenz interpretieren lassen (für einen Überblick siehe Klinzing und Rupp 2008). In der beruflichen Praxis finden validierte Fremd- oder Selbstbeurteilungsverfahren allerdings kaum Anwendung (Solga 2005). Die Evaluation von Maßnahmen zur Förderung der Präsentationskompetenz konzentriert sich auf selbstentwickelte Instrumente, die vorrangig affektive (z.B. Zufriedenheit der Trainingsteilnehmenden) und wissensbezogene Kriterien (z. B. Ausbau von präsentationsbezogenem Wissen) erfassen, während eine systematische Beurteilung von Veränderungen des Präsentationsverhalten (z.B. verbesserter Blickkontakt) oder die Messung organisationaler Effekte (z.B. gesteigerter Projekterfolg durch verbesserte Präsentationen von Zwischenergebnissen beim Kunden) selten Berücksichtigung finden (vgl. Blickle 2019; Solga 2005).

\section{Zusammenfassung und nächste Schritte}

Der Beitrag bietet einen differenzierten Überblick zur Diagnostik von Präsentationskompetenz, die sich als multidimensionales Konstrukt konzeptualisieren lässt. Ziel der Diagnostik ist es, Fach- und Führungskräfte sowie angehende Expert*innen bei der Einschätzung und Entwicklung ihrer Präsentationskompetenz zu unterstützen, wobei das präsentationsbezogene Wissen, die Kompetenzüberzeugungen und Zielorientierungen sowie das Präsentationsverhalten anhand Selbst- und Fremdeinschätzungsverfahren erfasst werden können. Im Arbeits- und Organisationskontext liegen bisher nur wenig methodisch ausgereifte empirische Studien vor, die Ansätze zur Förderung von Präsentationskompetenz untersuchen und/oder entsprechende Instrumente für den Einsatz im Kontext von Personalauswahl und -entwicklung konzipiert und validiert haben (Blickle 2019; Klinzing und Rupp 2008). Daher fokussiert der vorliegende Beitrag auf Instrumente, die mehrheitlich im Rahmen (hoch)schulischer Förderangebote entwickelt wurden und sich vorrangig an Personengruppen mit geringer Präsentationserfahrung wie Jugendliche und junge Erwachsene richten.

Allerdings lassen sich diese Förderangebote und die zugehörigen Diagnosetools unter Berücksichtigung der Charakteristika des jeweiligen Berufsfeldes vom Bildungs- auf den Arbeitskontext übertragen, da sich die Anforderungen an Präsentierende und die Grundausrichtung der Präsentationsaufgaben in beiden Settings deutlich überlappen (z. B. Blickle 2019; Hoffman et al. 2015; Preiser und Turashvili 2016; van Ginkel et al. 2015a). Beispielsweise zeigen sich hohe Überschneidungen in den betrachteten Facetten der Präsentationskompetenz sowie den Beurteilungsdimensionen für Schüler*innen, Studierende und Bewerber*innen in Assessment-Centern (Bartram 2005; Freimuth und Sieland 2013; Obermann 2018; Ruth et al. 2021; Schuler 2006). 
Vom sekundären über den tertiären Bildungsbereich hin zum Unternehmenskontext nimmt allerdings die Komplexität der Präsentationsaufgaben, die Bandbreite an Präsentationsinhalten und die Heterogenität der Zuhörergruppen $\mathrm{zu}$.

Im Rahmen der Personalauswahl und bei Potenzialanalysen erlauben die Instrumente ein standardisiertes Vorgehen, um eine erhöhte Nachvollziehbarkeit und Vergleichbarkeit des Diagnoseergebnisses zu gewährleisten. Im Hinblick auf Aus- und Weiterbildung lassen sich Ziele und Inhalte für konkrete Fördermaßnahmen spezifizieren, wenn im Vorfeld der Stand und die Qualität des präsentationsbezogenen Wissens, Kompetenzüberzeugungen und Zielorientierungen oder das Präsentationsverhalten evaluiert werden. Die Erfassung des präsentationsbezogenen Wissens bietet beispielsweise die Möglichkeit, Schulungsinhalte gezielt am Vorwissen der Teilnehmenden auszurichten, um theoretische Schwerpunkte im Verlauf anzupassen oder den Trainingserfolg auf der Ebene des Wissens zu überprüfen. Dies bezieht sich beispielsweise auf die Tiefe der theoretischen Inhalte zu den Mechanismen, Herausforderungen und Grenzen von sprachlichen Vergleichen. Verfahren zur Erfassung von präsentationsbezogenen Kompetenzüberzeugungen eignen sich, um Personengruppen mit Förderbedarf zu identifizieren und das Ausmaß der erzielten Veränderung durch den Besuch eines Präsentationstrainings zu bestimmen (Ringeisen et al. 2019b). Fremdbeurteilungsbögen wie das TIP helfen, die Angemessenheit des gezeigten Präsentationsverhaltens $\mathrm{zu}$ beurteilen, die gezeigte Leistung verschiedener Personen miteinander zu vergleichen, Bewertungskriterien transparent zu machen und Feedback zu geben, um Stärken und Entwicklungspotenziale der Präsentierenden abzuleiten.

Trotz dieser praktischen Implikationen ist weitere Forschung hinsichtlich der Definition und Förderung von Präsentationskompetenz notwendig. Das in Abb. 1 visualisierte Strukturmodell, das zwischen kompetenzkonstituierenden Merkmalen der Präsentierenden und verhaltensbasierter Präsentationskompetenz differenziert, ist empirisch zu überprüfen. Ferner besteht hoher Bedarf, die skizzierten Annahmen zur stufen- und entwicklungsbezogenen Differenzierung der Präsentationskompetenz zu validieren. In Anlehnung an das Vorgehen von Bartram (2005) ließe sich eine empirische Überprüfung der vorgeschlagenen Kompetenzmodelle realisieren. Dabei können - basierend beispielsweise auf Datensätzen aus dem hochschulischen oder betrieblichen Kontext - sowohl variablenzentrierte Verfahren wie Strukturgleichungsmodellierung als auch personenzentrierte Verfahren wie die Latent Class Analysis oder die Latent Transition Analysis einen wichtigen Beitrag zur Überprüfung der dargestellten Modelle liefern (Blömeke et al. 2015; Howard und Hoffman 2018). Variablenzentrierte Verfahren eignen sich, um die Gültigkeit der strukturellen Beziehungen zwischen den Facetten der Präsentationskompetenz und deren Ausdifferenzierung, wie in Abb. 1 skizziert, zu untersuchen. Personenzentrierte Verfahren helfen, bestimmte Subgruppen wie Präsentierende mit unterschiedlichen Kompetenzniveaus zu identifizieren (vgl. Geldmacher 2010) oder eine Ausdifferenzierung der Kompetenzstruktur im Sinne eines Entwicklungsmodells abzubilden. Parallel empfiehlt es sich, die Validität der vorgestellten Instrumente im Arbeits- und Organisationskontext systematisch und fortlaufend $\mathrm{zu}$ überprüfen, vor allem für ausgewählte Subgruppen wie Präsentierende mit einem hohen Kompetenzniveau.

Ergänzend wird empfohlen, das Konstrukt Präsentationskompetenz unter Einbeziehung weiterer Wissenschaftsdisziplinen multiperspektivisch zu reflektieren. Dies erfordert unter anderem die enge Zusammenarbeit der Disziplinen Deutschdidaktik, Rhetorik sowie Psychologie, Pädagogik, Sprach- und Sprechwissenschaft. Im Rahmen eines interdisziplinären Vorgehens ließe sich beispielsweise die Expertise der Sprachwissenschaft zur Modulation der Stimme um die rhetorischen, psychologischen und sprechwissenschaftlichen Perspektiven zur Stimme ergänzen, um so gemeinsame Forschungsfragen abzuleiten/zu untersuchen und um eine differenzierte theoretische Verortung dieser Subdimension in einen Gesamtrahmen der Präsentationskompetenz zu ermöglichen. Eine konzeptionelle Weiterentwicklung betrifft auch die theoretisch abgeleitete Unterteilung der einzelnen Verhaltenspotenziale und beispielsweise die Ausdifferenzierung einzelner Facetten der Präsentationskompetenz, wie der Körpersprache oder des Sprachgebrauchs. 


\section{Anhang}

Tab. 1 Fremdbeurteilungsbogen zur Einschätzung des Präsentationsverhaltens (Ringeisen et al. 2019b; Schickel und Ringeisen 2020), der in Anlehnung an De Grez et al. (2009) entwickelt wurde

\begin{tabular}{ll}
\hline Facetten des Präsentationsverhaltens & Indikatoren \\
\hline Gliederung & Präsentation wird gegliedert in Einleitung, Hauptteil und Schluss/Fazit/Ausblick \\
& Abzug: Gliederung undeutlich \\
Freies Formulieren & Inhalte werden frei formuliert vorgetragen \\
& Ungünstig: Vortrag ist abgelesen/auswendig gelernt \\
Blickkontakt & Vortragender hält Blickkontakt mit dem Publikum \\
& Ungünstig: Blick schweift ab, Blick zur Wand/zum Beamer etc \\
Körperhaltung & Dem Publikum zugewandte, offene und lockere Haltung, aufrechter Stand \\
& Ungünstig: Vom Publikum abgewandt, verkrampfte/hölzerne Haltung \\
Gestik/Mimik & Einsatz der Hände und der Mimik unterstützt die Wirkung des Vortrags \\
& Ungünstig: Hände werden in den Hosentaschen versteckt bzw. verschränkt, keine Mimik, Hampeln/ \\
Zappeln, Fuchteln der Hände & Angemessenes Tempo, Einsatz von Betonung, deutliche, modulierende Aussprache (Betonung), keine \\
Sprache & Füllwörter (z. B. Ähhs, genau) \\
& Ungünstig: zu schnell/zu langsam, zu leise, keine Betonung, Füllwörter \\
Sinsatz rhetorischer Mittel (z. B. rhetorische Frage/n, allgemeine oder persönliche Anekdote/n, Meta- \\
pher/n, direkte Publikumsansprache, etc.) \\
Ungünstig: keine rhetorischen Mittel \\
Zeitvorgaben werden eingehalten
\end{tabular}


Funding Open Access funding enabled and organized by Projekt DEAL.

Open Access Dieser Artikel wird unter der Creative Commons Namensnennung 4.0 International Lizenz veröffentlicht, welche die Nutzung, Vervielfältigung, Bearbeitung, Verbreitung und Wiedergabe in jeglichem Medium und Format erlaubt, sofern Sie den/die ursprünglichen Autor(en) und die Quelle ordnungsgemäß nennen, einen Link zur Creative Commons Lizenz beifügen und angeben, ob Änderungen vorgenommen wurden.

Die in diesem Artikel enthaltenen Bilder und sonstiges Drittmaterial unterliegen ebenfalls der genannten Creative Commons Lizenz, sofern sich aus der Abbildungslegende nichts anderes ergibt. Sofern das betreffende Material nicht unter der genannten Creative Commons Lizenz steht und die betreffende Handlung nicht nach gesetzlichen Vorschriften erlaubt ist, ist für die oben aufgeführten Weiterverwendungen des Materials die Einwilligung des jeweiligen Rechteinhabers einzuholen.

Weitere Details zur Lizenz entnehmen Sie bitte der Lizenzinformation auf http://creativecommons.org/licenses/by/4.0/deed.de.

\section{Literatur}

Aristoteles (2002). Rhetorik (C. Rapp, Übers.). Akademie Verlag. (Original-Werk veröffentlicht um ca. 335 v. Chr.)

Bachmann, G. (2009). Zielorientierungen und aktuelle Motivation: Eine Integration im Kontext des selbstregulierten Lernens. Doctoral dissertation. Frankfurt am Main: Johann Wolfgang Goethe-Universität. Retrieved from https://d-nb.info/100298078x/34

Backlund, P.M., \& Morreale, S.P. (2015). Communication competence: Historical synopsis, definitions, applications, and looking to the future. In A.F. Hannawa \& B.H. Spitzberg (Hrsg.), Communication Competence (Bd. 22, S. 11-38). Berlin: De Gruyter.

Bartram, D. (2005). The great eight competencies: a criterion-centric approach to validation. The Journal of Applied Psychology, 90(6), 1185-1203. https://doi.org/10.1037/0021-9010.90.6.1185.

Berkemeier, A. (2006). Präsentieren und Moderieren im Deutschunterricht. Baltmannsweiler: Schneider-Verl. Hohengehren.

Blickle, G. (2019). Personalentwicklung. In F. W. Nerdinger, G. Blickle, \& N. Schaper (Hrsg.), Springer-Lehrbuch. Arbeits- und Organisationspsychologie: Mit 32 Tabellen (Bd. 89, S. 325-355). Berlin: Springer. https://doi.org/10.1007/978-3-662-56666-4_19.

Blömeke, S., Gustafsson, J.-E., \& Shavelson, R.J. (2015). Beyond dichotomies: competence viewed as a continuum. Zeitschrift für Psychologie, 223(1), 3-13. https://doi.org/10.1027/2151-2604/ a000194.

Böhme, K. (2015). Didaktik der Rhetorik: Grundriss einer Didaktik der persuasiven Kommunikation. In J. Knape (Hrsg.), Neue Rhetorik 21 (S. 1-339). Berlin: Weidler Buchverlag.

Borman, W.C., Bryant, R. H., \& Dorio, J. (2010). The measurement of task performance as criteria in selection research. In J.L. Farr \& N. T. Tippins (Hrsg.), Handbook of employee selection. Hoboken: Taylor and Francis.

Boser, J., Hansen, M., \& Preiser, S. (2014). Präsentationsfertigkeiten von Studierenden fördern - Evaluation eines Seminarkonzepts. In M. Krämer, U. Weger, \& M. Zupanic (Hrsg.), Psychologiedidaktik und Evaluation X. PsychOpen. https://doi.org/10.23668/ psycharchives.970.

Braun, E., \& Hannover, B. (2011). Gelegenheiten zum Kompetenzerwerb in der universitären Lehre. Zeitschrift Für Entwicklungspsychologie Und Pädagogische Psychologie, 43(1), 22-28. https:// doi.org/10.1026/0049-8637/a000029.

Braun, E., Gusy, B., Leidner, B., \& Hannover, B. (2008). Das Berliner Evaluationsinstrument für selbsteingeschätzte, studentische
Kompetenzen (BEvaKomp). Diagnostica, 54(1), 30-42. https:// doi.org/10.1026/0012-1924.54.1.30.

Brünner, G. (2013). Vermittlungsstrategien in Gesundheitssendungen: Die Rolle von Metaphern, Vergleichen und anderen Verfahren der Veranschaulichung. In K. Birkner \& O. Ehmer (Hrsg.), Veranschaulichungsverfahren im Gespräch (S. 18-43). Mannheim: Verlag für Gesprächsforschung.

Bucher, H.-J. (2012). Multimodalität - ein universelles Merkmal der Medienkommunikation: Zum Verhältnis von Medienangebot und Medienrezeption. In H.-J. Bucher \& P. Schumacher (Hrsg.), Interaktionale Rezeptionsforschung (S. 51-82). Wiesbaden: Springer. https://doi.org/10.1007/978-3-531-93166-1_2.

Bucher, H.-J., Krieg, M., \& Niemann, P. (2010). Die wissenschaftliche Präsentation als multimodale Kommunikationsform. Empirische Befunde zu Rezeption und Verständlichkeit von Powerpoint-Präsentationen. In H.-J. Bucher, T. Gloning, \& K. Lehnen (Hrsg.), Neue Medien - neue Formate: Ausdifferenzierung und Konvergenz in der Medienkommunikation. Interaktiva, Schriftenreihe des Zentrums für Medien und Interaktivität, Gießen, (Bd. 10, S. 375-406). Frankfurt am Main: Campus.

Buchholz, N. (2009). Handlungsorientiertes Training oder selbstorganisiertes Lernen? Eine Evaluationsstudie zum Erwerb studienbezogener Kompetenzen zu Projektarbeit und Präsentation. In M. Krämer, S. Preiser, \& K. Brusdeylins (Hrsg.), Psychologiedidaktik und Evaluation VII (S. 347-358). Aachen: Shaker.

Buchholz, N., \& Eberle, J. (2003). Ausbildung von Studierenden zu Trainern sozialer Kompetenzen - Entwicklung bereichsspezifischer Selbstwirksamkeitsüberzeugungen. In G. Krampen \& H. Zayer (Hrsg.), Psychologiedidaktik und Evaluation IV (S. 365-386). Bonn: Deutscher Psychologen Verlag.

Chan, V. (2012). Teaching oral communication in undergraduate science: are we doing enough and doing it right? Journal of learning design, 4(3), 71-79. https://doi.org/10.5204/jld.v4i3.82.

Corno, L., Cronbach, L. J., Kupermintz, H., Lohman, D. F., Mandinach, E. B., Porteus, A. W., \& Talbert, J.E. (2002). The educational psychology series. Remaking the concept of aptitude: extending the legacy of Richard E. Snow. Mahwah: Lawrence Erlbaum.

De Grez, L., Valcke, M., \& Roozen, I. (2009). The impact of goal orientation, self-reflection and personal characteristics on the acquisition of oral presentation skills. European Journal of Psychology of Education, 24(3), 293-306. https://doi.org/10.1007/BF03174762.

De Grez, L., \& Valcke, M. (2010). Learning and instruction of oral presentation skills. In L.E. Kattington (Hrsg.), Education in a competitive and globalizing world. Handbook of curriculum development (S. 177-208). New York: Nova Science.

Dwyer, K. K., \& Davidson, M. M. (2012). Is public speaking really more feared than death? Communication Research Reports, 29(2), 99-107. https://doi.org/10.1080/08824096.2012.667772.

Elliot, A. J., \& Murayama, K. (2008). On the measurement of achievement goals: critique, illustration, and application. Journal of Educational Psychology, 100, 613-628. https://doi.org/10.1037/ 0022-0663.100.3.613.

Erpenbeck, J., v. Rosenstiel, L., Grote, S., \& Sauter, W. (Hrsg.). (2017). Handbuch Kompetenzmessung: Erkennen, verstehen und bewerten von Kompetenzen in der betrieblichen, pädagogischen und psychologischen Praxis (3. Aufl.). Stuttgart: Schäffer-Poeschel.

Freimuth, J., \& Sieland, J. (2013). Vorträge und Präsentationen. In W. Sarges (Hrsg.), Management-Diagnostik (4. Aufl. S. 668-676). Göttingen: Hogrefe.

Gätje, O., Krelle, M., Behrens, U., \& Grundler, E. (2016). Präsentieren als literale Kompetenz? Leseforum Schweiz. Literalität in Forschung und Praxis, (1). http://www.forumlecture.ch/ myUploadData/files/2016_1_Gaetje_et_al.pdf

Geldmacher, M. (2010). Präsentationskompetenz im gymnasialen Deutschunterricht. Ziele, Inhalte und Methoden. Hohengehren: Schneider. 
Herbein, E. (2017). Public speaking training as an enrichment program for elementary school children: Conceptualization, evaluation, and implementation. Doctoral dissertation. Tübingen: Universität, Universitätsbibliothek. https://doi.org/10.15496/ publikation-17711.

Herbein, E., Golle, J., Tibus, M., Schiefer, J., Trautwein, U., \& Zettler, I. (2018). Fostering elementary school children's public speaking skills: a randomized controlled trial. Learning and Instruction, 55, 158-168. https://doi.org/10.1016/j.learninstruc.2017.10.008.

Herdel, T., \& Preiser, S. (2018). Trainingskompetenzen - Validierung eines Trainer-Selbstkonzept-Fragebogens und Evaluation eines Trainings. In M. Krämer, S. Preiser, \& K. Brusdeylins (Hrsg.), Psychologiedidaktik und Evaluation XII. PsychOpen. (S. 317-323). https://doi.org/10.23668/psycharchives.1053.

Hochschulrektorenkonferenz und Kultusministerkonferenz (2017). Qualifikationsrahmen für deutsche Hochschulabschlüsse. https:// www.kmk.org/fileadmin/Dateien/pdf/PresseUndAktuelles/2017/ 2017-03-30_BS_170216_Qualifikationsrahmen.pdf. Zugegriffen: 24. Apr. 2021

Hoffman, B.J., Kennedy, C.L., LoPilato, A.C., Monahan, E.L., \& Lance, C.E. (2015). A review of the content, criterion-related, and construct-related validity of assessment center exercises. Journal of Applied Psychology, 100(4), 1143-1168. https://doi. org/10.1037/a0038707.

Howard, M.C., \& Hoffman, M.E. (2018). Variable-centered, person-centered, and person-specific approaches. Organizational Research Methods, 21(4), 846-876. https://doi.org/10.1177/ 1094428117744021.

John, M. (2007). Assessment-Center. In M. John (Hrsg.), Eignungsdiagnostik in der Personalarbeit: Grundlagen, Methoden, Erfahrungen (S. 231-252). Düsseldorf: Symposion.

Kliche, O. (2013). Von ne, kennen Sie ja bis zu ganz blöd mal gesprochen: kontextuelle Anschlussverfahren von Metaphern in Begriffserklärungen von Medizinstudierenden. In K. Birkner \& O. Ehmer (Hrsg.), Veranschaulichungsverfahren im Gespräch (S. 44-65). Mannheim: Verlag für Gesprächsforschung.

Klinzing, H. G., \& Rupp, A. (2008). How to become more effective in discussions? Four studies. Annual meeting of the American Educational Research Association, New York. https://files.eric.ed. gov/fulltext/ED501445.pdf

Knape, J. (2000). Was ist Rhetorik? Stuttgart: Reclam.

Knape, J. (2005). The Medium is the Massage? Medientheoretische Anfragen und Antworten der Rhetorik. In J. Knape (Hrsg.), Medienrhetorik (S. 17-39). Tübingen: Attempto.

Knape, J. (2013). Textleistung. Eine moderne rhetorische Kategorie, erprobt am Beispiel mittelalterlicher Chronistik. In K. Ridder \& S. Patzold (Hrsg.), Die Aktualität der Vormoderne: Epochenentwürfe zwischen Alterität und Kontinuität. Europa im Mittelalter, (Bd. 23, S. 135-159). Berlin: Akademie-Verl.

Kramer, O., \& Gottschling, M. (2012). Rhetorische Situation. In G. Ueding \& W. Jens (Hrsg.), Historisches Wörterbuch der Rhetorik (Bd. 10, S. 1126-1132). Berlin: De Gruyter.

Kramer, O., \& Malaka, R. (Hrsg.). (2014). Jugend Präsentiert: Handbuch für Multiplikatoren. Heidelberg: Klaus Tschira Stiftung.

Krause, D.E., Gebert, D., \& Thornton, G.C. (2007). Interkulturelle Unterschiede in der Assessment Center-Anwendung. In H. Schuler (Hrsg.), Wirtschaftspsychologie. Assessment Center zur Potenzialanalyse (S. 359-378). Göttingen: Hogrefe.

Kuhn, C., Zlatkin-Troitschanskaia, O., Pant, H. A., \& Hannover, B. (2016). Valide Erfassung der Kompetenzen von Studierenden in der Hochschulbildung. Zeitschrift für Erziehungswissenschaft, 19(2), 275-298. https://doi.org/10.1007/s11618-016-0673-7.

Lipphardt, C. (2019). Untersuchung zur Trainierbarkeit verbaler Evidentialisierungstechniken in naturwissenschaftlichen Schülerpräsentationen als Problem der modernen Rhetorik. Doctoral dissertation. Tübingen: Universität Tübingen. https://doi.org/10.15496/ publikation-37026.
Lipphardt, C., Ruth, F., \& Kramer, O. (2017). Präsentation im MINTUnterricht. Jugend präsentiert als Projekt zur Entwicklung kommunikativer Kompetenz. In U. Trautwein \& M. Hasselhorn (Hrsg.), Begabungen und Talente. Jahrbuch der pädagogisch-psychologischen Diagnostik. Tests und Trends, (Bd. 15, S. 229-244). Göttingen: Hogrefe.

Lobin, H. (2007). Textsorte „Wissenschaftliche Präsentation”“ - Textlinguistische Bemerkungen zu einer komplexen Kommunikationsform. In B. Schnettler \& H. Knoblauch (Hrsg.), PowerpointPräsentationen: Neue Formen der gesellschaftlichen Kommunikation von Wissen (S. 67-82). Konstanz: UVK.

Lobin, H. (2009). Inszeniertes Reden auf der Medienbühne: Zur Linguistik und Rhetorik der wissenschaftlichen Präsentation. Interaktiva. Bd. 8. Frankfurt: Campus.

Morreale, S.P., Moore, M., Surges-Tatum, D., \& Webster, L. (2007). The competent speaker speech evaluation form (2. Aufl.). Washington, D.C: National Communication Association.

Morreale, S.P., Spitzberg, B.H., \& Barge, J. K. (2013). Communication: motivation, knowledge, skills (3. Aufl.). New York: Peter Lang Publishing.

Mulder, M. (2014). Conceptions of professional competence. In S. Billett, C. Harteis, \& H. Gruber (Hrsg.), International handbook of research in professional and practice-based learning (S. 107-137). Dordrecht: Springer Netherlands. https://doi.org/ 10.1007/978-94-017-8902-8_5.

Obermann, C. (2018). Assessment Center. Entwicklung, Durchführung, Trends. Wiesbaden: Springer Gabler.

Obermann, C., Höft, S., \& Janke, O. (2008). Was machen die DAX100Unternehmen? Die große AkAC-Studie 2008. In Arbeitskreis Assessment Center e. V. (Hrsg.), Diagnostische Kompetenz: entwickeln und anwenden: Dokumentation zum 7. Deutschen Assessment-Center-Kongress 2008 (S. 266-295). Lengerich: Pabst Science Publ.

Preiser, S., \& Turashvili, T. (2016). Wissenschaftskommunikation und Experten-Laien-Kommunikation: Kompetenzerwerb durch Trainingsseminare an deutschen und georgischen Universitäten. In M. Krämer, S. Preiser, \& K. Brusdeylins (Hrsg.), Psychologiedidaktik und Evaluation XI (S. 93-100). Aachen: Shaker. https:// doi.org/10.23668/psycharchives.986

Rahn, B., \& Moser, K. (2000). Beiträge verschiedener Prädiktoren zur Kriteriumsvalidität eines Assessment Centers. German Journal of Human Resource Management: Zeitschrift Für Personalforschung, 14(2), 177-190. https://doi.org/10.1177/ 239700220001400203.

Ringeisen, T., \& Bürgermeister, A. (2015). Fostering students' selfefficacy in presentation skills: the effect of autonomy, relatedness and competence support. In K. Moore, S. Howard, \& P. Buchwald (Hrsg.), Stress and anxiety: application to schools, well-being, coping and internet use (S. 77-87). Berlin: Logos.

Ringeisen, T., Lichtenfeld, S., Becker, S., \& Minkley, N. (2019a). Stress experience and performance during an oral exam: the role of self-efficacy, threat appraisals, anxiety, and cortisol. Anxiety, Stress, and Coping, 32(1), 50-66. https://doi.org/10.1080/ 10615806.2018.1528528.

Ringeisen, T., Rohrmann, S., Bürgermeister, A., \& Tibubos, A.N. (2019b). Assessing self-efficacy in presentation and moderation skills. European Journal of Psychological Assessment, 35, 564-576. https://doi.org/10.1027/1015-5759/a000423.

Rubin, R. B., \& Morreale, S. P. (1996). Setting expectations for speech communication and listening. New Directions for Higher Education, 1996(96), 19-29. https://doi.org/10.1002/he.36919969604.

Ruth, F. (2020). Linking rhetoric and educational research: The assessment and promotion of secondary school students' presentation competence. Doctoral dissertation. Tübingen: Universität, Universitätsbibliothek. https://doi.org/10.15496/publikation-51121.

Ruth, F., Herbein, E., Fauth, B., Trautwein, U., \& Kramer, O. (2021). Towards a psychometrically sound assessment of students' pre- 
sentation competence: The development of the Tübingen Instrument for Presentation Competence (TIP). [Manuskript in Vorbereitung]. Universität Tübingen.

Schaeper, H., \& Spangenberg, H. (2008). Absolventenbefragungen - Erfassung relevanter Kompetenzen für Studium und Beruf. In N. Jude, J. Hartig, \& E. Klieme (Hrsg.), Kompetenzerfassung in pädagogischen Handlungsfeldern. Theorien, Konzepte, Methoden. Bildungsforschung, (Bd. 26, S. 161-175). Berlin: BMBF.

Schickel, M., \& Ringeisen, T. (2020). What predicts students' presentation performance? Self-efficacy, boredom and competence changes during presentation training. Current Psychology, 105(1), 150. https://doi.org/10.1007/s12144-020-01090-8.

Schickel, M., Minkley, N., \& Ringeisen, T. (2021). Performance during presentations: A question of perceived challenge and threat? [Manuskript eingereicht zur Publikation]. Berlin School of Economics and Law.

Schmidt-Rathjens, C. (2007). Anforderungsanalyse und Kompetenzmodellierung. In H. Schuler \& K.-H. Sonntag (Hrsg.), Handbuch der Arbeits- und Organisationspsychologie. Handbuch der Psychologie, (Bd. 6, S. 592-601). Göttingen: Hogrefe.

Schnettler, B., \& Knoblauch, H. (2007). Die Präsentation der „Wissensgesellschaft“". Gegenwartsdiagnostische Nachüberlegungen. In B. Schnettler \& H. Knoblauch (Hrsg.), Powerpoint-Präsentationen: Neue Formen der gesellschaftlichen Kommunikation von Wissen (S. 267-283). Konstanz: UVK.

Schreiber, L.M., Paul, G.D., \& Shibley, L.R. (2012). The development and test of the public speaking competence rubric. Communication Education, 61(3), 205-233. https://doi.org/10.1080/ 03634523.2012.670709.

Schuler, H. (2006). Arbeits-und Anforderungsanalyse. In H. Schuler (Hrsg.), Lehrbuch der Personalpsychologie (S. 45-68). Göttingen: Hogrefe.

Smith, C. M., \& Sodano, T.M. (2011). Integrating lecture capture as a teaching strategy to improve student presentation skills through self-assessment. Active Learning in Higher Education, 12(3), 151-162. https://doi.org/10.1177/1469787411415082.

Solga, M. (2005). Evaluation der Personalentwicklung. In J. Ryschka, M. Solga, \& A. Mattenklott (Hrsg.), Praxishandbuch Personalentwicklung. Instrumente, Konzepte, Beispiele (S. 293-324). Wiesbaden: Gabler.

Sonntag, K., \& Schmidt-Rathjens, C. (2005). Anforderungsanalyse und Kompetenzmodelle. In P. Gonon, F. Klauser, R. Nickolaus, \& R. Huisinga (Hrsg.), Kompetenz, Kognition und neue Konzepte der beruflichen Bildung (S. 55-66). Wiesbaden: VS.

Sonntag, K.-H., \& Schaper, N. (2006). Förderung beruflicher Handlungskompetenz. In K.H. Sonntag (Hrsg.), Personalentwicklung in Organisationen (S. 230-311). Göttingen: Hogrefe.

Spencer, L. M., \& Spencer, S. M. (1993). Competence at work: Models for superior performance. New York: Wiley.

Staton, A.Q., \& Tomlinson, S.D. (2001). Communication education outreach in elementary school classrooms. Southern Communication Journal, 66(3), 211-223. https://doi.org/10.1080/ 10417940109373200 .

Treutner, D. (2016). Optimierung eines Kommunikationsseminars mit Videofeedback anhand von Videoannotation. In M. Krämer, S. Preiser, \& K. Brusdeylins (Hrsg.), Psychologiedidaktik und Evaluation XI (S. 119-125). Aachen: Shaker. https://doi.org/10. 23668/psycharchives. 989 .

Ueding, G., \& Steinbrink, B. (2011). Grundriß der Rhetorik: Geschichte Technik Methode (5. Aufl.). Stuttgart: J.B. Metzler..

Van Ginkel, S., Gulikers, J., Biemans, H., \& Mulder, M. (2015a). Towards a set of design principles for developing oral presentation competence: a synthesis of research in higher education. Educational Research Review, 14, 62-80. https://doi.org/10.1016/j. edurev.2015.02.002

Van Ginkel, S., Gulikers, J., Biemans, H., \& Mulder, M. (2015b). The impact of the feedback source on developing oral presentation competence. Studies in Higher Education, 42(9), 1671-1685. https://doi.org/10.1080/03075079.2015.1117064.

Weinert, F. E. (Hrsg.). (2002). Leistungsmessungen in Schulen (2. Aufl.). Weinheim: Beltz.

Wyßuwa, F., \& Beier, F. (2013). Beispielerzählungen und Szenarioentwicklung in der Weiterbildung als Veranschaulichungen von Wissen und Relevanzen. In K. Birkner \& O. Ehmer (Hrsg.), Veranschaulichungsverfahren im Gespräch (S. 133-155). Mannheim: Verlag für Gesprächsforschung.

Yurong, S. (2015). An overview of the development of research on public speaking course from 2004 to 2013. Chinese Journal of Applied Linguistics. https://doi.org/10.1515/cjal-2015-0027.

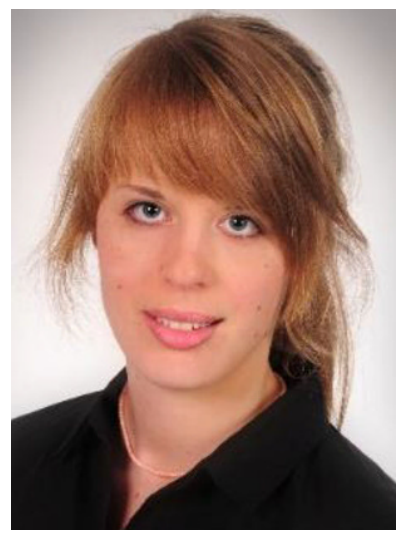

Evelin Herbein Dr. Evelin Herbein ist Diplom-Psychologin und Sprecherzieherin (Univ.). Sie leitet das Referat 42 „Begleitforschung, Forschungskooperation" am Institut für Bildungsanalysen Baden-Württemberg (IBBW) in Stuttgart. Davor war sie bis 2019 als wissenschaftliche Mitarbeiterin am Hector-Institut für Empirische Bildungsforschung an der Universität Tübingen tätig. Ihre inhaltlichen Schwerpunkte und Forschungsinteressen liegen auf der Konzeption, Implementation und Wirksamkeitsprüfung von Interventionen im Bildungskontext. Ein Hauptfokus bildet dabei die Entwicklung, Erfassung und Förderung von Präsentationskompetenz und Unterrichtsqualität.

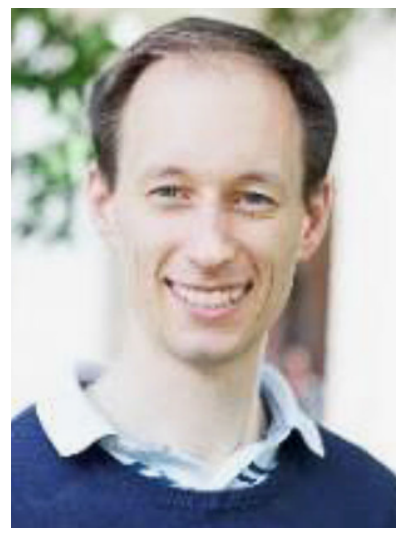

Fabian Ruth Dr. Fabian Ruth ist akademischer Mitarbeiter an der Forschungsstelle Präsentationskompetenz am Seminar für Allgemeine Rhetorik der Universität Tübingen. Dort ist er für die wissenschaftliche Begleitforschung des Projektes Jugend präsentiert zuständig. In seiner Forschungstätigkeit setzt er sich mit Fragen der empirischen Bildungsund Rhetorikforschung auseinander, insbesondere mit der Messung von Präsentationskompetenz und der Wirksamkeit von Präsentationstrainings. Fabian Ruth gibt Trainings zur Präsentationskompetenz sowie Methodik und Didaktik für unterschiedliche Zielgruppen wie Schüler*innen, Lehrkräfte und Lehrende allgemein. 


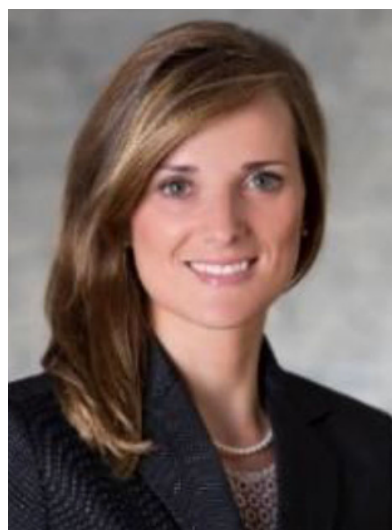

Carmen Lipphardt Dr. Carmen Lipphardt ist akademische Mitarbeiterin an der Forschungsstelle Präsentationskompetenz am Seminar für Allgemeine Rhetorik der Universität Tübingen, wo sie mitverantwortlich ist für die präsentationsbezogene Forschung und die Weiterentwicklung des Trainingsangebots von Jugend präsentiert. Carmen Lipphardt konzipierte die Ausbildung für Juniortrainer*innen, in der Rhetorikstudierende während ihres Studiums zu Präsentationstrainer*innen ausgebildet werden, und leitet die Jugend präsentiertTrainerakademie. Ihre Forschungsinteressen auf dem Gebiet der Präsentationskompetenz liegen im Bereich der sprachlichen Gestaltung, insbesondere der Erzeugung von Anschaulichkeit in MINT-Präsentationen. Carmen Lipphardt hat zudem zahlreiche praktische Erfahrungen als Trainerin und Beraterin in den Bereichen Präsentation, Kommunikation und Moderation gesammelt.

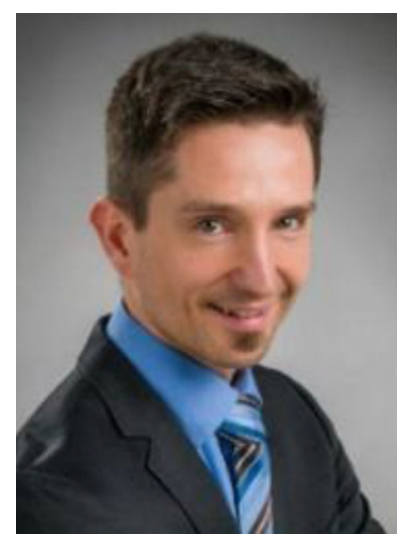

Tobias Ringeisen Herr Dr. Tobias Ringeisen ist Professor für Angewandte Psychologie an der Berlin School of Economics and Law. Er beschäftigt sich in Forschung und Praxis mit der Förderung und Diagnostik von berufsrelevanten Kompetenzen im jungen Erwachsenenalter. Sein besonderes Interesse gilt dem Zusammenspiel von Persönlichkeitsmerkmalen, Lernprozessen und begleitenden Emotionen sowie Leistungsindikatoren. Weiterhin beschäftigt er sich mit der Bedeutung von Diversität in beruflichen und lernbezogenen Settings, insbesondere (inter)kultureller Diversität. Zu den genannten Themenkomplexen hat er mehr als 80 Publikationen in Form von Büchern, Zeitschriftenartikeln, Buchkapiteln und Fragebögen veröffentlicht. Zudem setzt er sich in Anwendungsprojekten mit Wirtschaft und Verwaltung für eine enge Verzahnung von Theorie und Praxis ein. Unter anderem war er als Personalentwickler, Consultant und Trainer in mehreren Unternehmen und Bundesverwaltungen sowie als wissenschaftlicher Mitarbeiter am Boston College, USA, und der Bergischen Universität Wuppertal tätig. Seit 2020 fungiert Herr Ringeisen als Präsident der Stress and Anxiety Research Society (STAR Society; https://star-society.org/). 